\title{
Numerical analysis on the generation, propagation and interaction of solitary waves by a Harmonic Polynomial Cell Method
}

\author{
Tong, Chao; Shao, Yanlin; Hanssen, Finn-Christian W.; Li, Ye; Xie, Bin; Lin, Zhiliang
}

Published in:

Wave Motion

Link to article, DOI:

10.1016/j.wavemoti.2019.01.007

Publication date:

2019

Document Version

Peer reviewed version

Link back to DTU Orbit

Citation (APA):

Tong, C., Shao, Y., Hanssen, F-C. W., Li, Y., Xie, B., \& Lin, Z. (2019). Numerical analysis on the generation, propagation and interaction of solitary waves by a Harmonic Polynomial Cell Method. Wave Motion, 88, 34-56. https://doi.org/10.1016/j.wavemoti.2019.01.007

\section{General rights}

Copyright and moral rights for the publications made accessible in the public portal are retained by the authors and/or other copyright owners and it is a condition of accessing publications that users recognise and abide by the legal requirements associated with these rights.

- Users may download and print one copy of any publication from the public portal for the purpose of private study or research.

- You may not further distribute the material or use it for any profit-making activity or commercial gain

- You may freely distribute the URL identifying the publication in the public portal 


\section{Accepted Manuscript}

Numerical analysis on the generation, propagation and interaction of solitary waves by a Harmonic Polynomial Cell Method

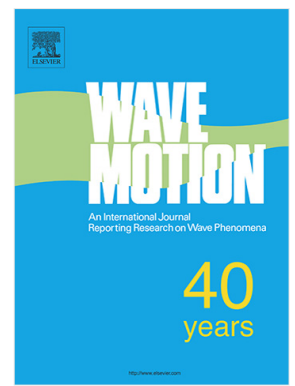

Chao Tong, Yanlin Shao, Finn-Christian W. Hanssen, Ye Li, Bin Xie, Zhiliang Lin

PII:

S0165-2125(18)30361-5

DOI: https://doi.org/10.1016/j.wavemoti.2019.01.007

Reference: $\quad$ WAMOT 2326

To appear in: Wave Motion

Received date: 5 September 2018

Revised date: 10 January 2019

Accepted date : 13 January 2019

Please cite this article as: C. Tong, Y. Shao, F.-C.W. Hanssen et al., Numerical analysis on the generation, propagation and interaction of solitary waves by a Harmonic Polynomial Cell Method, Wave Motion (2019), https://doi.org/10.1016/j.wavemoti.2019.01.007

This is a PDF file of an unedited manuscript that has been accepted for publication. As a service to our customers we are providing this early version of the manuscript. The manuscript will undergo copyediting, typesetting, and review of the resulting proof before it is published in its final form. Please note that during the production process errors may be discovered which could affect the content, and all legal disclaimers that apply to the journal pertain. 


\title{
Numerical Analysis on the Generation, Propagation and Interaction of Solitary Waves
}

\section{by a Harmonic Polynomial Cell Method}

\author{
Chao Tong ${ }^{1}$, Yanlin Shao ${ }^{2}$, Finn-Christian W. Hanssen ${ }^{3}, \mathrm{Ye} \mathrm{Li}^{1,4,5,{ }^{*}}$ Bin Xie $^{1,4}, \mathrm{Z}_{\text {.liliang }}{ }^{\text {in }}{ }^{1,4}$
}

1. School of Naval Architecture, Ocean \& Civil Engineering, Shanghai Jiao Tong U ،vt sity, Shanghai, 200240, China

2. Department of Mechanical Engineering, Technical University of Denmark

3. Centre for Autonomous Marine Operations and Systems (AMOS), Norv oo in Un versity of Science and Technology

4. Collaborative Innovation Center for Advanced Ship and Deep-Sea Exploratio. Shanghai Jiao Tong University, Shanghai 200240, China

5. Key Laboratory of the Ministry of Education, Shanghai Jiao Tong Unı ersity, ' hanghai 200240, China

\section{Abstract}

A numerical wave tank based on the Harmonic Polynomial Cell $\left(\mathrm{H}_{\imath} \mathcal{C}\right) \mathrm{m} \epsilon^{\mathrm{L}}, \mathrm{d}$ is created to study the generation, propagation and interaction of solitary waves. The HPC method has been prov $\mathrm{n}$ to be of high accuracy and efficiency in modelling of water waves, wave-wave and wave-structure interaction $w^{\text {thin }}$. 1e context of potential flow. An important

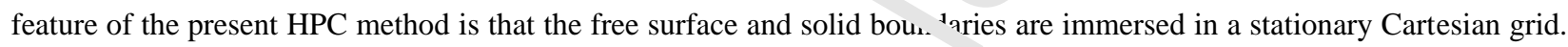
Solitary waves with $\sigma$, i.e. amplitude to water depth ratio, up t $\quad \cdots .0$ are generated by different methods. We demonstrate that the results based on the first-, third- and ninth-order method are - ss satisfactory than the fully-nonlinear method in generating solitary waves with $\sigma>0.4$. Additionally, both the ear' on and overtaking collision between two solitary waves are studied. In the investigation of the phase shifts after + - heac on collision, our window model successfully explain the main reason why Su \& Mirie [1]'s third-order approximatioı or 'œe uniform phase shifts is inconsistent with Chen \& Yeh's [2] experimental results and Craig et al.'s [3] fully ....... numerical results. For the overtaking collision of solitary waves, the collision process and the phase shifts are numerı "ly analyzed. Our present result also confirms Craig et al.'s [3] category of the overtaking collision.

Keywords: solitary wave; wave generation; wa e propą ttion; wave interaction; Harmonic Polynomial Cell method.

\section{Introduction}

Since discovered by Scott Russel' r4], solitaıy waves have played an important role in modelling tsunamis near the shore. In order to better understan 4 the $\mathrm{h}_{\text {. }}$-hanism of solitary waves, there have been considerable studies on their behaviors. Among these research $f, t_{1}$. essential aspect is how to generate solitary waves accurately. There exist various methods of solitary wave gent. tio $^{\prime}$ in wave flumes [5-11], among which the piston-type wave maker is the most commonly used in experime is. Cons. 'ering that the prescribed velocity of the wave paddle shall equal the uniform horizontal velocity of the $\mathrm{w}$ er $\mathrm{f} \mathrm{rticl}^{\prime} s$ on the wave maker boundary, Goring [8] derived the motion of a piston-type wave maker. By applying Boussinesq $r 1$. J solitary wave solution, Goring's method has been primarily used in experiments to study solitary waves in ne last c 'cades [13-15]. However, it was observed by Grilli \& Svendsen [16] in a numerical study that Goring's method on. ' perfo' ned well for solitary waves of smaller amplitude, but the amplitude of a generated solitary wave was slightly s naller than the desired value and attenuated continuously during propagation for higher waves, in spite of neglecting watc viscosi $\%$. In order to generate solitary waves as accurate as possible, Katell \& Eric [9] carried out experiments to genel a. . ulitary waves by using a paddle motion law from Rayleigh [17], and they observed that the features of gen, "ate' waves were in close agreement with those of desired ones. However, Wu et al. [18] pointed out that the boundary outs. 'rt decay coefficient used in Katell \& Eric [9] was smaller than the value based on Boussinesq's [12] solution. More water volume has been consequently pushed forward so that the wave amplitude was higher than the designed value in their numerical study. Having noticed the transient behaviors of solitary waves adjacent to the wave paddle in the process of wave generation, Malek-Mohammadi \& Testik [10] proposed a new methodology by assuming that the wave speed is time-dependent at the wave paddle during wave generation. They concluded that solitary waves generated 
through their methodology were less dispersive to retain a permanent form. Based on Goring's paddle movement formula, Wu et al. [18] carried out numerical experiments by using different solitary wave solutions. They found that Goring's formula using the ninth-order solution of Fenton [19] could extend the applicability of Goring's method to a relative higher wave amplitude. Therefore, a modified Goring's method was proposed and laboratorial experimf ts were also carried out later by Wu et al. [11] in a water flume to validate the modified Goring's method.

It is known that the maximum run-up during a head-on collision of two solitary waves $i^{\prime}$ n1 'er than the sum of their initial wave amplitudes [20]. Maxworthy [21] carried out experiments to investigate the run- $\iota_{\star}$, ar $\star$ the phase shifts after the head-on collision of two identical solitary waves. It was confirmed that the wave profile rec hed a. aximum run-up greater than twice the initial wave amplitude and both waves suffered a time delay after the int ... tion. . . : \& Mirie [1] proposed a third-order perturbation method to analyze the head-on collision of two arbitrary-amr itudf solı ry waves and obtained the solutions for maximum run-up, phase shifts and the secondary trailing waves. In a later nu nerical analysis, Mirie \& Su [22] found that solitary waves lost energy to the trailing waves causing reduction of vave ar. vlitude. Fenton \& Rienecker [23] numerically studied the head-on collision based on Fourier series. They pointed $c$ 't that a' hough the maximum run-up was consistent with Su \& Mirie's [1] third-order approximation and Chan \& Stref s 241 numerical result, the third-order theory seemed unable to predict the phase shifts. The measurement of the phase s ift r $_{\text {is }}$ st ongly dependent on the measurement location due to the change of the wave speed after the interaction. Renc ${ }^{\prime} r d e^{\prime} \cdot$. [25] in their experiments observed an amplitude decay after the reflection of a solitary wave from a vertical wall. $\mathbf{1}_{\mathbf{1}}$ ? wave reflection was also investigated by Cooker et al. [26]. They discovered that both amplitude and energy lu occ $c$ after the reflection due to the dispersive trailing wavetrain. It was also found that the run-up process was show - than the run-down process. In other words, the asymmetry of the process could have resulted in the observed. $4 u 1 n g$ waves. Craig et al. [3] systematically studied the head-on collision and overtaking collision of two solitary waves by sc. ing the Euler equations for water-wave problems. They analyzed the residual produced by the wave-wave inter. tic \& and estimated the energy carried away by the trailing waves. Laboratorial experiments on the head-on collision $f$ two litary waves were carried out by Chen \& Yeh [2] using the laser induced fluorescence (LIF) technique with a mo tag - method to provide precise measurement of free surface profile. Chen et al. [27] also showed detailed flow f u - a locity, acceleration, vorticity and velocity-gradient tensor related to the head-on collision.

For the overtaking collision of two differen+-1itary waves, Lax [28] theoretically proposed a category of the KdV overtaking interaction based on the amplitude ttio of th two solitary waves. The same notation was also employed in the experiments of Weidman \& Maxworthy [29] Zabu 'v - Kruskal [30] simulated a large solitary wave overtaking a smaller one and found that the solitary waves ret ned neir initial shapes after the interaction except for a spatial phase change. Mirie \& Su [22] observed that the trailirg $w$. es $b$, ind the smaller wave after the interaction are much smaller than those in a head-on collision. Fenton \& Rier -ker [23] carried out a simulation of an overtaking collision of two solitary waves with amplitude ratio of 3.142 and fuund thin the category proposed by Lax [28] was not able to explain their numerical results. Similar discrepancy was iso ibserved by Craig et al. [3] in their numerical and experimental study. Hence, a modification of Lax's [28] categ ${ }^{\prime}$ ' h's been proposed by Craig et al. [3]. Fenton \& Rienecker [23] and Craig et al. [3] also discovered that the higher wa' 2 woula ' 'ghtly grow in amplitude but the opposite for the smaller one after the interaction. Gardner et al. [31] showed nat + te or staking collision between two solitary waves were elastic, where the term 'elastic' means that solitary waves will $\ldots .9$. 1 their wave form and velocity except for phase shift after the collision. Sachs [32] showed that overtakin̨ collisic of solitary waves was elastic up to the second-order approximation. Zou \& Su [33]

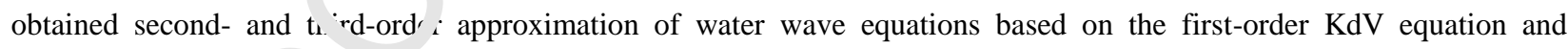
confirmed that the svertaking collision was elastic up to second order approximation. Their third-order numerical results were also able to e olain th $\epsilon$ dispersive trailing waves behind the smaller solitary wave after the interaction.

There exist man, ...erent numerical methods that can model solitary waves in the literature. It has been a strong tradition to use he wuidary Element Method [34,35] to model the fully-nonlinear water waves in the frame of potential flow. However, the hottleneck of the conventional Boundary Element Method is that both CPU time and computer memory requirement increase quadratically with the number of unknowns. Under this circumstances, Shao \& Faltinsen [36] newly proposed a potential-flow field solver named the HPC method, which has been proven to be highly efficient and accurate for 2D fully-nonlinear potential flow problems. Lately the HPC method was extended to 3D by Shao \& Faltinsen [37] and it was also demonstrated to be of high efficiency and accuracy in some marine applications, such as liquid sloshing in tanks, 
nonlinear wave diffraction and wave propagation over various topographies. Additionally, the HPC method has been advanced in many aspects in recent years. Fredriksen et al. [38] coupled a Navier-Stokes solver with the HPC method to study the water behavior in the moonpool of a ship. Liang et al. [30] used the HPC method along with a domain decomposition strategy to treat the singular flow at sharp corners. They also solved lifting probler · of a hydrofoil by using a double-layer nodes technique. Zhu et al. [40] and Hanssen et al. [45] applied an immersed frec surfac. ctrategy to the 2D HPC method. Hanssen et al. [41] created a nonlinear numerical wave tank by the $2 \mathrm{D}$ HPC $\mathrm{m}$ ' no, adopting two strategies to track the free surface, i.e. immersed boundary (IB) and multigrid. Ma et al. [42] gave a c 'ail $d$ and systematic analysis on the local and global properties of the 2D HPC method. After a comparison of various g. ' 'ting s. 'tegies, they concluded

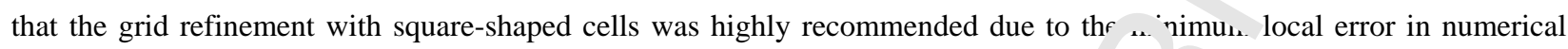
calculations. Bardazzi et al [43] extended the original 3D method to a generalized $\mathrm{H} C \mathrm{C}$-thu, which was confirmed the $4^{\text {th }}$ order accuracy when the method was applied in solving Poisson equation and even 1 . dealing with cases with singular solutions. Wang \& Faltinsen [44] applied Cartesian grids with an adaptive local $r$ inemei ' in the HPC method to accurately represent the highly-deformed free surface boundary and complicated body geo. etries. the method makes it possible to solve highly-nonlinear wave-wave and wave-structure interaction problems -. . the prunging breakers and water entry of solid objects, very accurately and efficiently.

In the present study, a fully-nonlinear wave tank is created by the $2 \mathrm{D} .{ }^{\mathrm{D}} \mathrm{C} \mathrm{m}^{+1} \mathrm{Jd}$. The free surface, which is treated as an immersed boundary in a structured Cartesian grid, is tracked by a set of mark, "s moving only in vertical direction.

The paper is organized as follows: In Section 2 we give an introducı $\urcorner$ the present 2D HPC method and the treatments of free surface conditions in both the background grid and the wave naker grid. In Section 3, after we compare the numerically generated solitary waves by using different solutio. valıation of the numerical results against laboratorial experiments is also presented. In Section 4, the head-on collision of $\triangle 1$ itary waves is investigated with the focus on the phase shifts after the interaction. Additionally, the overtaking ol' slun is also studied and the category of the overtaking interaction is discussed.

\section{Mathematical consideration}

\subsection{D Harmonic Polynomial Cell method}

A numerical water wave tank is defined $a^{-1}$ he origin of a global Cartesian coordinate system with axes $(x, z)$ is located at the midpoint of the tank, on the mer 1 water 1 vel. A local body-fixed coordinate system $x^{\prime} O^{\prime} z^{\prime}$ with the origin at the bottom of the wave paddle is also introd 'ced, hic' is applied in the wave maker fixed reference frame in Sec. 2.3. A sketch of the wave tank and the coordinate yste as are given in Figure. 1.

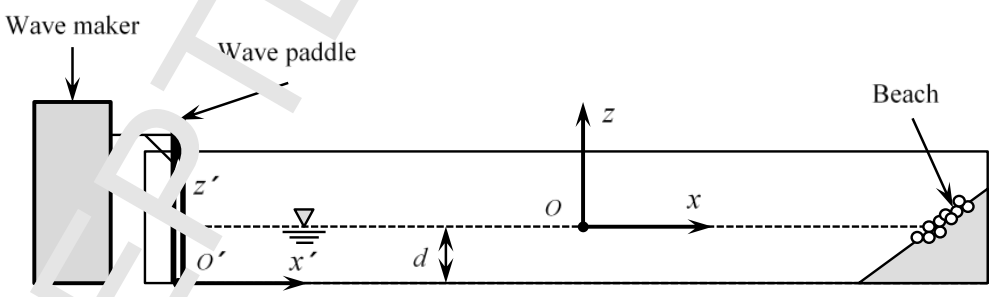

Figure 1. Sketch of a 2D water $w$ v tank with a piston-type generator. Both the global coordinate system $x O z$ and the local body-fixed coordinate system $x^{\prime} O^{\prime} z^{\prime}$ are introduced.

The fluid is as umed to be incompressible, inviscid, and the flow is irrotational, which can be described by the Laplace equation:

$$
\Delta \varphi(x, z)=0
$$

The whole co. 'putational domain can be discretized by quadrilateral overlapping cells as shown in Figure. 2. Each cell consists of eight boundary nodes with local indices 1-8 and a central node with local index 9. We introduce a local cell coordinate system with the origin at the central node, namely, 


$$
\left\{\begin{array}{l}
\zeta=x-x_{9} \\
\xi=z-z_{9}
\end{array}\right.
$$

where $(x, z)$ are global coordinates and $\left(x_{9}, z_{9}\right)$ are the global coordinates of the central node.

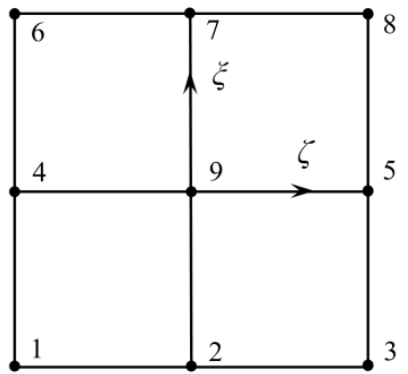

Figure 2. Sketch of a cell with local node indices and the ${ }^{1}$ sal cell coordinate system.

According to Shao \& Faltinsen [36], the velocity potential $\varphi$ it an ${ }^{r^{\prime}}$ itrary location within a cell can be approximated by the linear superposition of velocity potential $\varphi_{i}$ of the eight $b_{2}$ indary nodes

$$
\varphi(\zeta, \xi)=\sum_{i=1}^{8}\left[\sum_{j=1}^{8} c_{j, i} f_{j}(\zeta, \xi)\right] \varphi_{i} .
$$

Here $f_{j}(\zeta, \xi)$ are the eight harmonic polynomials given by ${ }^{\circ}$ ๆо \& Faltinsen [36]: $f_{1}(\zeta, \xi)=1 ; f_{2}(\zeta, \xi)=\zeta$; $f_{3}(\zeta, \xi)=\xi \quad ; \quad f_{4}(\zeta, \xi)=\zeta^{2}-\xi^{2} \quad ; \quad f_{5}(\zeta, \xi)=\zeta \xi \quad f_{6}(\zeta, \xi)=\zeta^{3}-3 \zeta \xi^{2} \quad ; \quad f_{7}(\zeta, \xi)=3 \zeta^{2} \xi-\xi^{3} \quad ;$ $f_{8}(\zeta, \xi)=\zeta^{4}-6 \zeta^{2} \xi^{2}+\xi^{4}$, where $c_{j, i}$ are elements of . invt e of the matrix [D] whose elements are $d_{j, i}=f_{j}\left(\zeta_{i}, \xi_{i}\right)$.

Since all these harmonic polynomials satisfy the Lap ${ }^{1}$ nce equ tion everywhere in the fluid domain, the velocity potential described in Eq. (3) automatically satisfies the Laplace ey ation.

The velocity potential of the $9^{\text {th }}$ node in each cell can be further simplified as

$$
\left.\varphi_{9}=\varphi\left(\zeta_{9}=\right), \xi_{9}=0\right)=\sum_{i=1}^{8} c_{1, i} \varphi_{i} .
$$

This equation serves as a connectivity equ r. Ion empı yed for every node in the fluid domain (except for nodes along the boundaries of the numerical wave tank), $k \rightarrow$ au $\_$ear $\_$of them will be the central node of a particular cell. For grid nodes in the fluid, it is straightforward to impose continu. " of velocity potential in the fluid by Eq. (4). For points in the domain that do not coincident with grid nodes, the $v_{\text {. }}$ - ity potential can be directly approximated by using Eq. (3) with local coordinate $\left(\zeta_{p}, \xi_{p}\right)$ in an appropriately chosen sell. For nodes along a Neumann boundary, the Neumann boundary conditions are fulfilled by taking the normal der vativ , of Eq. (3)

$$
\frac{\partial \varphi}{\partial}, \zeta, \xi=\sum_{i=1}^{8}\left[\sum_{j=1}^{8} c_{j, i} \nabla f_{j}(\zeta, \xi) \cdot \vec{n}(\zeta, \xi)\right] \varphi_{i},
$$

where $\vec{n}(\zeta, \xi)$ is the uni norma' vector of the Neumann boundary at the node $(\zeta, \xi)$.

\subsection{Free surface br andary conditions in the background grid}

In our study, in Imme sed Boundary (IB) method is used to handle the free surface conditions. The IB method was primarily combined $、$ 'th t' $^{\prime}$ 2D HPC method by Hanssen et al. [45] to impose body-boundary conditions on a solid body in an exterior flc v. 1 - ethod along with a free-surface tracking strategy was later used for imposing Dirichlet-type free-surface cond ${ }_{\imath}$ ons in a fully nonlinear water wave tank [41]. In our numerical experiments, the free surface is assumed to be calm at $t=0 \mathrm{~s}$. Ne technically represent the free surface by a set of markers, which are evenly distributed in horizontal direction. As illustrated in Figure. 3, the free-surface markers are only allowed to move freely along the vertical grid lines in the background Cartesian gird, so that the wave elevation is assumed as a single-value function. Each free-surface marker is restricted in the upper half area of the associated cell. The upper layer cells of the free surface are marked in gray in 
Figure. 3, which contain certain nodes above the free surface. Those nodes shall be considered as ghost nodes, whose velocity potentials are also unknown. Free surface conditions are enforced by applying Eq. (3) with local coordinates $\left(\zeta_{m}, \xi_{m}\right)$ of free surface markers. Since the grid refinement remain unchanged at any time step, the inverse of matrix [D] only needs to be determined once at the start of the simulation.

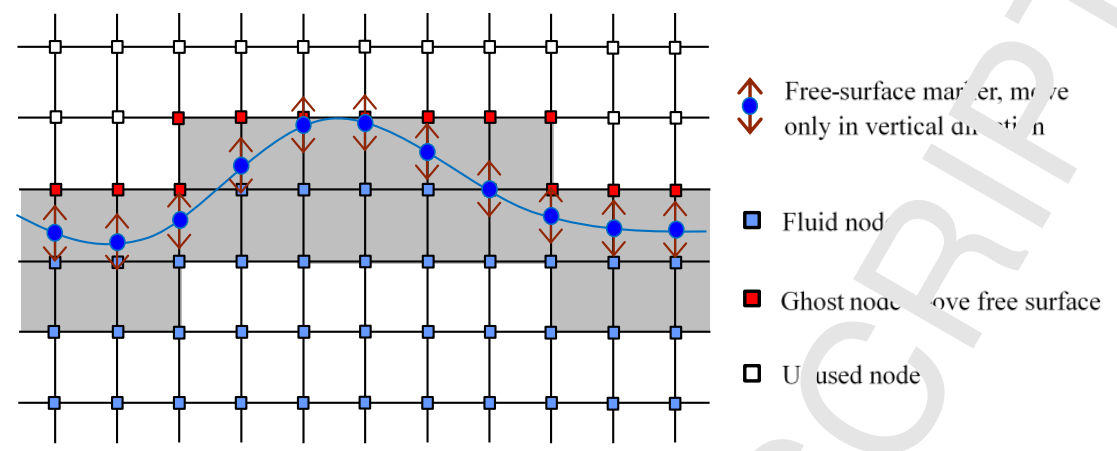

Figure 3. The free surface is treated as an immersed boundary in a str ctr ed b ckground grid, which is tracked by free-surface markers moving vertically as indicated by arrows. The cells $u$. $-d$ to $i \cdots$. ose free-surface conditions on are filled with gray.

In order to satisfy the fully nonlinear free surface conditions, th semi-Lagrangian method is adopted to describe kinematic and dynamic free surface conditions for a vertically $\mathrm{m}$. ug miliker with global coordinates $\left(x_{m}, z_{m}\right)$, namely

$$
\frac{D x_{m}}{D t}=0, \frac{D z_{m}}{D t}=\frac{\partial \varphi}{\partial z}-\frac{\partial \varphi}{\partial x} \frac{\partial \eta}{\partial x} \cdots \cdots_{n}:
$$

$$
\frac{D \varphi_{m}}{D t}=\frac{\partial \varphi}{\partial t}+\frac{\partial \eta}{\partial t} \frac{\partial \varphi}{\partial z}-v(x) \varphi
$$

$$
=-\frac{1}{2}\left|\left(\frac{\partial \varphi}{\partial x}\right)^{2}+\left(\frac{\partial \varphi}{\partial z}\right)^{2}\right|-g l_{l} \quad \frac{\partial_{l}}{\partial t} \frac{\partial \varphi}{\partial z}-v(x) \varphi,
$$

where $z=\eta(t, x)$ denotes the free surface elev . .1on, $g$ is the gravity acceleration and the term $v(x)$ is a damping coefficient that is used to absorb the energy of the wave at th an $r^{\prime}$ of the tank to minimize wave reflection from the downstream tank wall. More details of the damping zone an dar ping coefficients are referred in Greco [46] and Hanssen et al. [41]. In the study of the interaction and reflection of $s^{\prime}{ }^{\prime}$ ary $\mathrm{r}$ aves, $v(x)$ is set to be zero. Here we have used the semi-Lagrangian derivative $D / D t=\partial / \partial t+\vec{v}_{m} \cdot \nabla$, wher $\vec{v}_{n}=\left(0, \partial \kappa_{m} / \partial t\right)$. All spatial derivatives are calculated at the instantaneous position $\left(x_{m}, z_{m}\right)$ of a marker. The wave slop $\partial \eta, \nabla_{\boldsymbol{x}}$ is evaluated using a high-order finite difference scheme. We use an explicit fourth-order Runge-Kutta scheme $\ldots$ th e domain to integrate the free surface conditions.

\subsection{Free surface boundary cr aditions the wave-maker grid}

A physical piston-type vav $\mathrm{mak} r$ is simulated by introducing a local body-fitted grid on the right side of the wave paddle. This local grid overlaps $w^{\prime} \cdot h$.he background Cartesian grid, as depicted in Figure. 4. This local grid also slides over

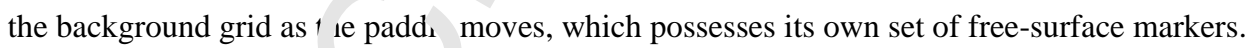

As described previc sly for he background grid, the free surface markers are only allowed to move along vertical grid lines in the global $r$ Jordin ${ }^{+}$e system. Similarly, markers in the wave maker grid are also only allowed to move along the $z^{\prime}$ axis in the wave $n$ tker fixe $\mid$ reference frame. The corresponding semi-Lagrangian free surface conditions for a marker in this reference frame ca... written as:

$$
\frac{D x_{m}^{\prime}}{D t}=0, \frac{D z_{m}^{\prime}}{D t}=\frac{\partial z_{m}^{\prime}}{\partial t}+\left(u_{\text {grid }}^{\prime}-\frac{\partial \varphi}{\partial x^{\prime}}\right) \frac{\partial \eta^{\prime}}{\partial x^{\prime}}
$$

$$
\frac{D \varphi_{m}}{D t}=-\frac{1}{2}\left|\left(\frac{\partial \varphi}{\partial x^{\prime}}\right)^{2}+\left(\frac{\partial \varphi}{\partial z^{\prime}}\right)^{2}\right|-g \eta^{\prime}+u_{\text {grid }}^{\prime} \frac{\partial \varphi}{\partial x^{\prime}}+\left(w_{\text {grid }}^{\prime}+\frac{\partial \eta^{\prime}}{\partial t}\right) \frac{\partial \varphi}{\partial z^{\prime}} .
$$

$u_{\text {grid }}^{\prime}$ and $w_{\text {grid }}^{\prime}$ are the horizontal and vertical velocity components of the grid point at a position $\left(x^{\prime}, z^{\prime}\right)$ related to the 


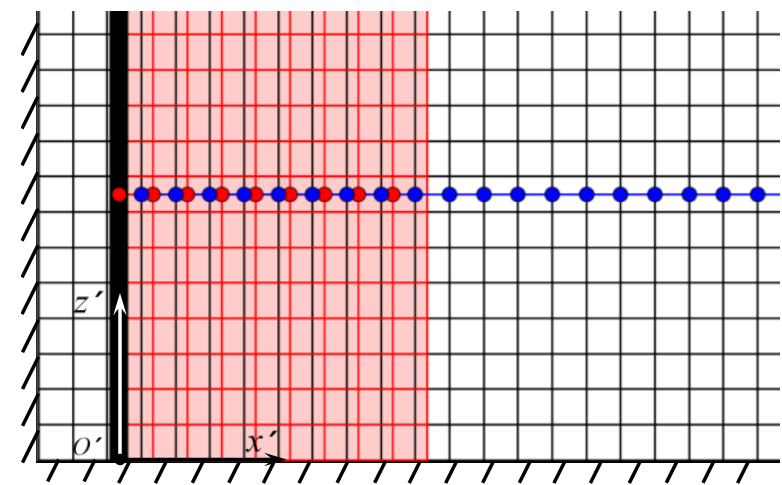

Free-surface mal, $\mathrm{I}$ background gria

- Free-surfa mak $s$ in wavema"er-fixu rid

Figure 4. Modeling of the piston-type wave maker with structured body-fixed gri The lo al grid fixed on the wave marker is marked in red while the background grid is marked in black.

By using overlapping grids, an important part is the exchange of inf and the background grid. The velocity potential of a grid point in the local gl ${ }^{1}$ can be interpolated from the background grid through:

$$
\varphi_{W M G}\left(x^{\prime}, z^{\prime}\right)=\sum_{i=1}^{8}\left[\sum_{j=1}^{8} c_{j, i} f_{j}(\zeta, \xi)\right], \Im_{G},
$$

where $\varphi_{W M G}$ and $\varphi_{B G G}$ are the velocity potential in the wave man $r$ gr 4 u. $d$ in the background grid, respectively. Since in the fluid domain the velocity potential of a particular point : iniqu regardless of the selected reference frame, the velocity potential of a grid point $(\zeta, \xi)$ in the wave maker grid can $b_{c} \mathrm{q}_{\mathrm{r}}$ 'xumated by a cell in the background grid that contains the node $\left(x^{\prime}, z^{\prime}\right)$, i.e. by applying Eq. (10). Correspondi. . . , . - n $\lambda_{+}$of the background grid in the overlapping region can also get the velocity potential from interpolation in the wave-man $r$ fitted grid:

$$
\left.\varphi_{B G G}(x, z)=\sum_{i=1}^{8}\left[\sum c_{j, i} f_{j} \zeta^{\prime}, \xi^{\prime}\right)\right]\left.\varphi_{i}\right|_{W M G} .
$$

This ensures a direct two-way couplin, bet een the regions in an implicit form.

All these boundary conditions togethe th $\mathbf{F}_{\mathbf{1}}$. (4) are combined to build a global linear algebraic matrix system for the velocity potential in the form of $\left[f\{\varphi\}=\left\{\iota_{f}\right.\right.$. The coefficient matrix $[A]$ is sparse with at most 9 nonzero entities in each row so that the equations can be solveu fficiently with standard solvers for sparse-matrix equations.

\section{Validation and verifica. on}

The numerical water wave ank Jaser on the HPC method presented in Section 2 will be verified and validated in this section through studies on colita, 'v ve generation and head-on collision of two solitary waves.

\subsection{Solitary wave gen ration}

Piston-type wave $m_{\iota}$ kers ha e been widely used to generate solitary waves. Wu et al. [18] compared the solitary waves generated by appl ..1g Bo'ıssinesq's [12] first-order solitary wave solution, Rayleigh's [17] solution, Grimshaw's [47] third-order solutio and Fe ton's [19] ninth-order solution to Goring's [8] paddle motion formula. However, the use of Goring's formula is th umpliance with the fluid particle velocity along the wave maker boundary, especially for highly nonlinear solita $\mathrm{v}$ W $v$ v. In that case, they were still unable to produce solitary waves of desired dimensionless amplitudes as $\sigma>0.4(\sigma=A / a \quad d$ is the water depth).

In this part, we use Clamond \& Dutykh's [48] fully nonlinear solitary wave solution as the wave maker boundary condition. The main difference between this method and Goring's method is that the wave maker is kept stationary in the process of generating waves. We only update fully nonlinear solution of the horizontal velocity along the wave maker boundary below the free surface, so that the method is named Fully Nonlinear (FNL) method. 
Primarily, the numerical experiments of solitary wave generation are conducted to assess the convergence of the spatial and temporal discretization for $\sigma=0.4$ by the FNL method. The wave tank is set to be $L=40 \mathrm{~m}$, the tank height $H=2 \mathrm{~m}$ and the water depth $d=1 \mathrm{~m}$. Table 1 gives the information of three grid refinements. $(\Delta x / d, \Delta z / d)$ ard $\left(n_{x}, n_{z}\right)$ denote length and number of grid elements in horizontal and vertical direction, respectively.

Table 1. Grid refinements for the wave tank domain.

\begin{tabular}{ccccc}
\hline Case & $\Delta x / d$ & $n_{x}$ & $\Delta z / d$ & \\
\hline Coarse & 0.20 & 200 & 0.20 & $n$ \\
\hline Medium & 0.10 & 400 & 0.10 & 20 \\
\hline Fine & 0.0667 & 600 & 0.0667 & 20 \\
\hline
\end{tabular}

According to Clamond \& Dutykh's [48] fully nonlinear solution, the wave s] eed of $\mathrm{t}_{\mathrm{1}}$. solitary wave is C $=3.6892 \mathrm{~m} / \mathrm{s}$. To make sure the $C F L$ number $(C F L=C \Delta t / \Delta x)$ below unity, the non-dimensio. 11 tim step $\Delta \bar{t}=\Delta t \sqrt{g / d}=0.01$ is used for all simulations. Figure 5 depicts the free surface at $\bar{t}=t \sqrt{g / d}=37.5^{\prime} 9$ fr $\mathrm{u}$ : three grid refinements. The results obtained here using the fine and medium mesh strategy are almost coin : Nent whil the amplitude cannot reach the target value for the coarse mesh strategy. This indicates that the grid convergence $1 \mathrm{~s}$ shieved using the medium grid refinement. Similar tests for different time steps $(\Delta t=0.01 \mathrm{~s}, 0.02 \mathrm{~s}$ and $0.025 \mathrm{~s}) \mathrm{u} \cdot \mathrm{ng}$ the nedium grid refinement indicate that $\Delta t=$ $0.02 \mathrm{~s}$ is sufficient for the accuracy.

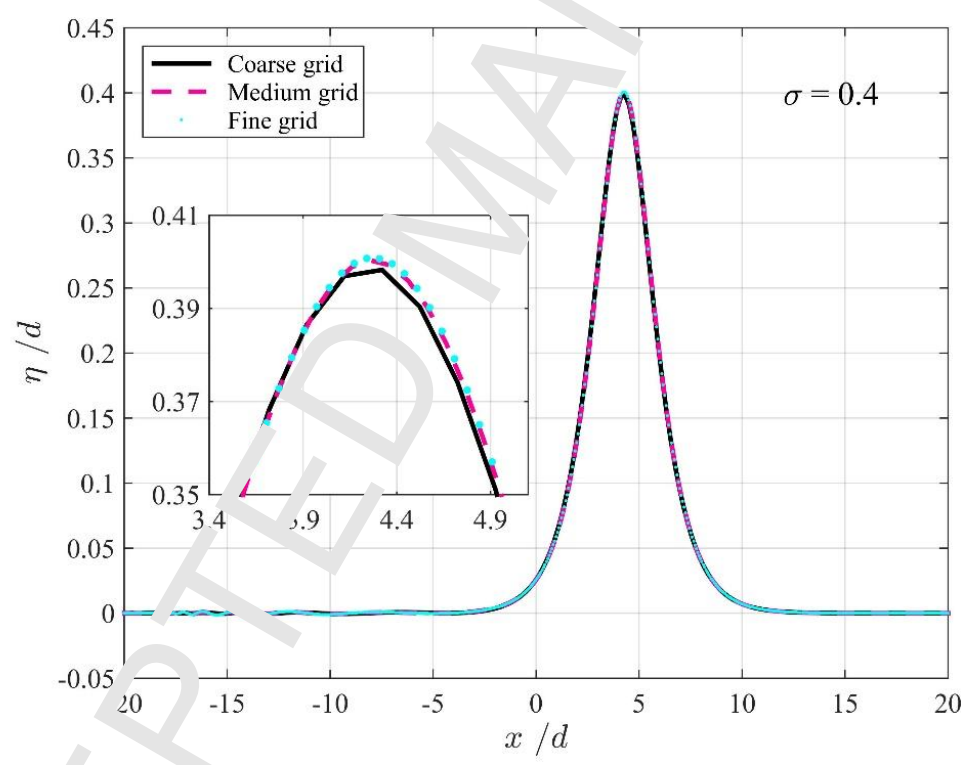

Figure 5 Fre surf $^{\prime}$ ce profiles of solitary wave with $\sigma=0.4$ for different grid refinements.

T ،ble 2. The list of numerical conditions of cases for solitary wave generation.

\begin{tabular}{ccc}
- Methor number & Method & Target wave amplitude $\sigma$ \\
\hline 1 & G1S & $0.2|0.4| 0.6$ \\
\hline 2 & G3S & $0.2|0.4| 0.6$ \\
\hline 3 & G9S & $0.2|0.4| 0.6$ \\
\hline 4 & FLN & $0.2|0.4| 0.6$ \\
\hline
\end{tabular}

To conform the accuracy of this method, we compare numerical results of the FNL method with those of Goring's method by using the first-, third-, and ninth-order solitary wave solution (Here, we call them G1S, G3S and G9S method for simplicity). We have to remark that the wave maker is only kept still when applying the FNL method to produce solitary waves, but not for Goring's method. 
The height of the numerical wave tank is $H=2 \mathrm{~m}$, the length is $L=80 \mathrm{~m}$ and the calm water depth is $d=1 \mathrm{~m}$. The whole domain is horizontally and vertically discretized into 600 and 30 elements, respectively. A time step $\Delta t=0.02 \mathrm{~s}$ is chosen in this simulation. The conditions of the cases are listed in Table 2.

Figure. 6(a), 6(b) and 6(c) compare snapshots of wave profiles at a time when the wave crest rives at $x / d=15$ for $\sigma=$ $0.2,0,4$ and 0.6, respectively. For the smaller solitary wave, i.e. $\sigma=0.2$, the figure shows good . reement of wave elevations among the G3S, G9S and FNL method. However, the wave amplitude based on the sis method fails to reach the target value and the trailing waves are more visible than those in other methods. As the wa a s nplitude increases to 0.4, G3S and G9S method's wave profiles (both the wave crest and trailing waves) are almost 'tentic. 'but the trailing waves are still nearly invisible for the FNL method's result. For a solitary wave of amplitude $9.6,1 \imath 3$ seen from Figure. 6(c) that the solitary wave generated by the FNL method can not only reach the desired $w$.ve a .plı..de but also propagate with minor dispersive trailing waves. But all the other three methods underestimate the max11. $m$ wave amplitude and they also introduce non-negligible trailing waves. There also exist clear phase difference, betwe $\eta$ Goring's method and the FNL method as can be seen in Figure. 6(c). Since the generated wave using the FN methe, is higher than that of Goring's method, the corresponding phase speed is also slightly larger. Figure. $6(\mathbf{d}$, snows temporal evolution of the maximum amplitude for the three cases. G1S method's result of the maximum ; nn' ude seems to first overpredict but then underpredict the wave height during propagation. This is especially appa. ${ }^{\prime} n t$ fo ${ }^{`}$ gher waves. This observation indicates that the dispersive effect in the G1S method is much stronger when comparea ith the other three methods. One can also notice that, for $\sigma \geq 0.4$, the maximum amplitude based on the G1S meth، ' seer s to be much lower than the target value. A similar discrepancy was also observed in Wu et al. [18] for $\sigma \geq 0.4$ by $u_{\text {. }}$ og the G1S method. Moreover, Wu et al. [18] and Hanssen et al. [41] have also used the G9S method to generate. "ltary waves of amplitude $\sigma=0.6$. As a comparison, the FNL method is proven to be able to generate solitary waves much clos to desired ones, even for waves with amplitude as high as $\sigma=0.6$.

(a)

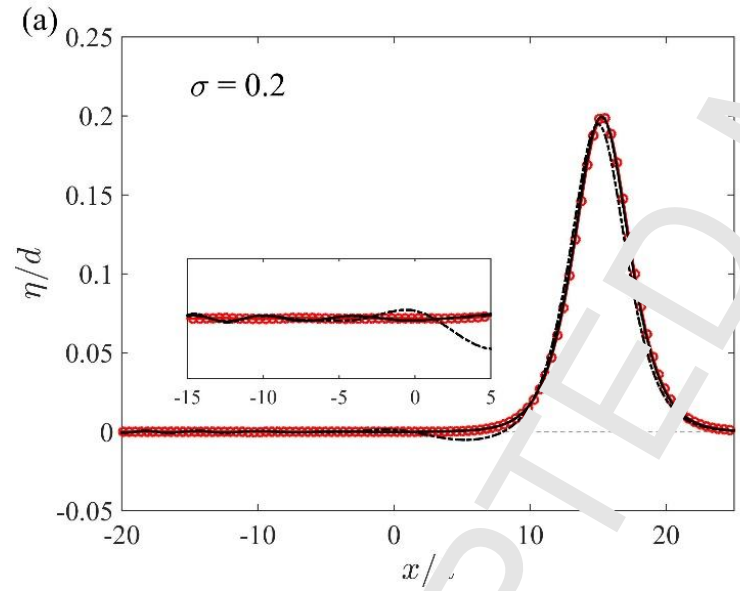

(c)

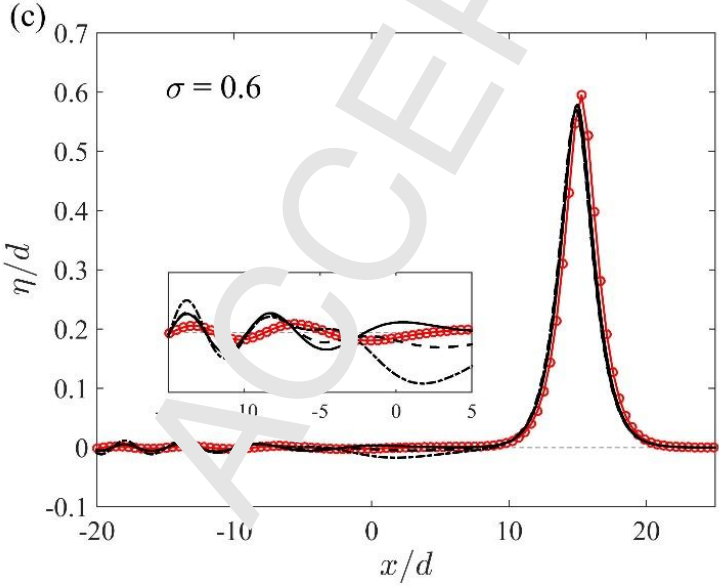

(b)

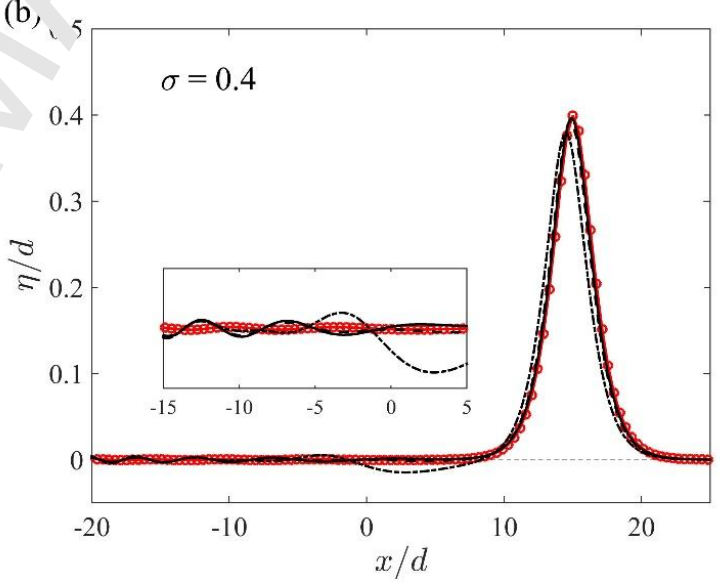

(d)

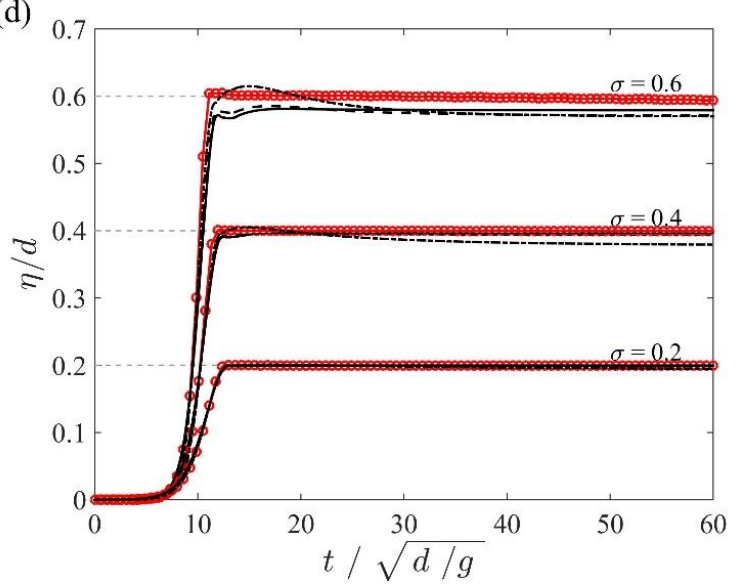

Figure 6. Subplots (a), (b) and (c) display snapshots of the wave profiles by using the G1S method (.- - - ) , the G3S method (- - ), the G9S method (— ) and the FNL method ( $-\circ$ ) for solitary wave of amplitude $\sigma=0.2,0.4$ and 0.6 . 
Subplot (d) shows temporal evolution of the maximum wave elevations for solitary wave of amplitude $\sigma=0.2,0.4$ and 0.6 .

The cause of the deviation between Goring's method and the FNL method is mainly because horizontal velocities of water particles are assumed uniform along the wave maker, while in the FNL method the full' nonlinear solution of horizontal velocity is directly imposed on the wave maker boundary. Therefore, the higher-ord · terms in solitary wave solutions become fairly important as $\sigma$ increases. Goring's paddle-motion formula ignores the iigher- - Jer effects, which eventually results in some dispersion errors, causing both trailing waves and reduction in wa c a. plitude. In this way, the FNL method seems superior when it comes to higher-amplitude solitary waves. This also ndi ates the wave-generation mechanism rather than the numerical method resulting in disparity of numerical results hen $u_{\text {. }}^{\text {` }}$ erent wave-generation methods are used.

Based on the comparisons presented above, the use of a piston-type wave maker' $y$ th $\mathrm{G}_{\mathrm{u}} \mathrm{m}$ method is accurate enough for solitary waves of amplitudes $\sigma \leq 0.4$, while the FNL method might be needed for muc.. higher waves. In Sec.3.2, we will compare our numerical results with experimental measurements where the pistc 1-type : vve maker is used. The largest $\sigma$ value considered therein is 0.4 .

\subsection{Head-on collision of solitary waves \\ 3.2.1 Wave run-up at a wall}

As for the reflection of solitary waves from a wall, He \& Kashiwagi [49] 1l. restigated the interaction of a solitary wave with a vertical elastic plate. Ertekin et al. [50] in their study mention ' tha' the reflection process is equivalent to the head-on collision between two identical solitary waves, so that we can . $_{\text {s }}$ - gine that there is an exactly upright wall located at the collision center during the head-on collision. In this part, ve Gys method is used for solitary wave generation. In present computations, a 2D domain with calm water depth $d=1 \mathrm{~m}$, tann 'eight $H=3 \mathrm{~m}$ and tank length $L=40 \mathrm{~m}$ is assumed. The whole domain is evenly discretized into 600 elements $\eta \mathrm{r}$,rızuntal direction and 75 elements along the vertical direction. A time step is set as $0.02 s$ in the time-domain si rlatio. A set of solitary waves with different normalized initial amplitudes, $A / d=0.1,0.15,0.2,0.25,0.3,0.35,0.4,0.45,0 . . \quad v_{2}=0.6$, are simulated. Figure. 7 presents the comparison of the maximum run-up among Su \& Mirie's [1] first-, s .... n. 1 third-order approximation, Cooker et al.'s [26] numerical results, the experimental results of Camfield \& Street [7] a. d Maxworthy [21]. According to Su \& Mirie's [1] third-order solution, the non-dimensional maximum run-up $\mathrm{c}^{-1}$ quantified in the form as:

$$
\sigma_{\max }=--+\frac{1}{2} \sigma^{2} \cdot \frac{3}{4} \sigma^{3} \text {. }
$$

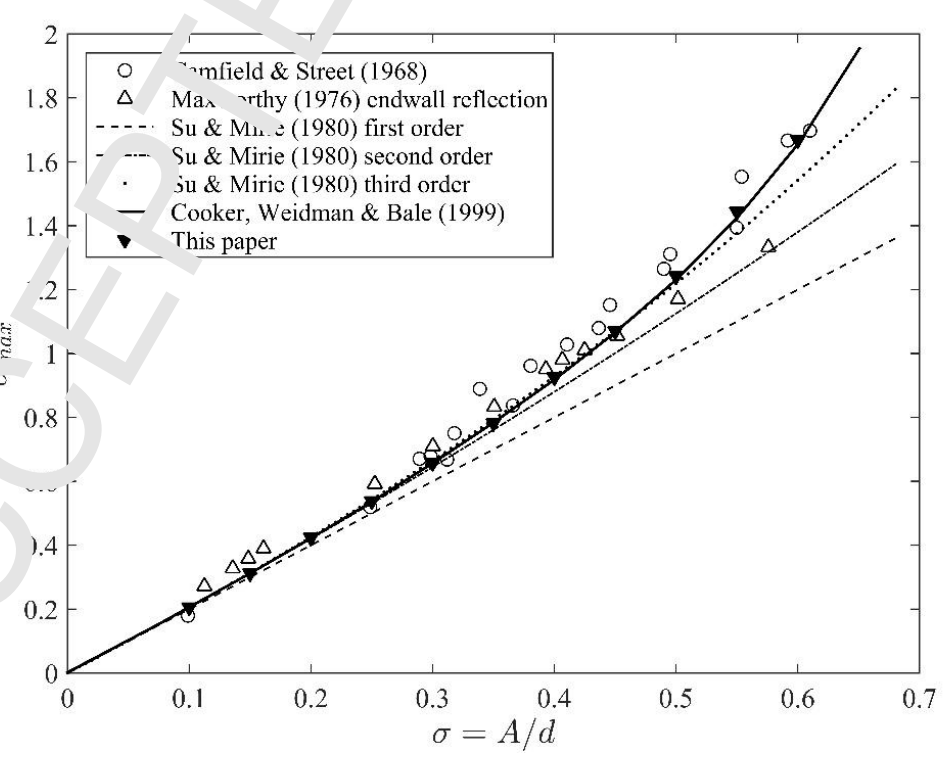

Figure 7. Maximum run-up versus incident wave amplitude.

From Figure. 7, it can be found that the results of the present HPC method are consistent with Su \& Mirie's [1] 
third-order results except for higher incident waves. Both the results of our calculation and Cooker et al. [26] appear to be obviously higher than Su \& Mirie's [1] third-order predictions when the incident wave amplitude is larger than 0.5, which indicates the importance of nonlinear terms higher than $3^{\text {th }}$ order. The fourth-order or even higher order solutions might, therefore, be required for predicting the maximum run-up in experiments. In our study, trailing wa as are created due to the use of the G9S method. Consequently, the wave amplitude will be lower than the target value. so elin. ate this effect, the wave maker signal has to be calibrated to generate the incident waves of desired amplitudes. J . ou or words, the input wave should be slightly higher than the desired one during wave generation. The same technique cr nmonly implemented for generating high-amplitude solitary waves in physical wave flumes. One can see from Fig $\cdots .7$ u. $^{+}$the present numerical results are in excellent agreement with Cooker et al.'s [26] results.

\subsubsection{Comparison with experimental results}

In this part, we validate our numerical model with experimental measurf nents c c Chen \& Yeh [2] on symmetric head-on collision of identical solitary waves with amplitudes $\sigma_{R}=\sigma_{L}=0.4$. Here, $\sigma=A_{R} / d$ and $\sigma_{L}=A_{L} / d$ denote non-dimensional amplitude of the right- and left-running wave, respectively igure. $\mathbf{o}$ shows wave crest trajectories of the present numerical results and experimental results with excellent agreemer. $C^{\prime}$,en $d$ Yeh [2] reported, in their study, that the phase speed of a solitary wave slowed down resulting from the reduch $、$ in a tude after the collision. However, from the plot of crest trajectories and the slopes of the linear fitted lines in Figur 8, it is clear that the wave speed of two solitary waves has slightly increased after the interaction, which is obvle cly $r$, ntradictory with the conclusion of Chen \& Yeh [2]. Similar to our report, Maxworthy [21] also mentioned, acc -ding to their experimental observation, that the reflected wave moved slightly faster than the incoming wave. spite of the attenuation in height. On the other hand, Byatt-Smith [20], Su \& Mirie [1] and Chan \& Street [24] conclude the unchanged wave amplitude after the head-on collision from either their theoretical predictions or numerical olv 'Ons. Fenton \& Rienecker [23] also noticed the increase of wave speed for a returned solitary wave from a vertic ' wall. They suggested that even though the crest elevation was actually reduced, the trough of the first trailing wave might av nlayed a significant role in increasing the 'effective wave height' (the vertical distance from the trailing troug. . 1 . . ain crest), which consequently results in the wave speed augment.

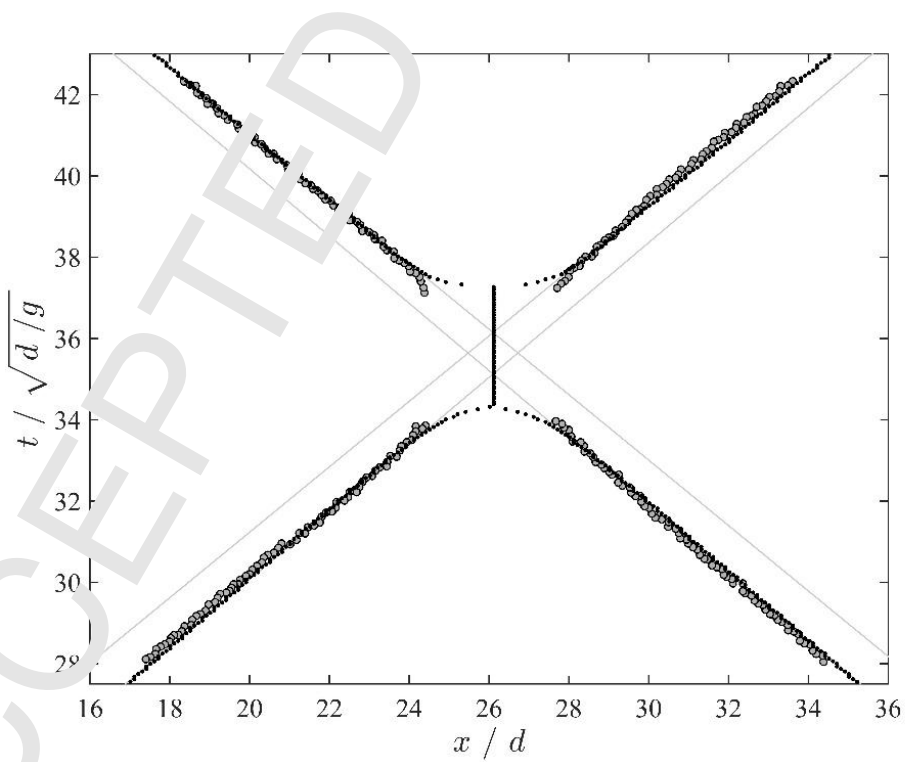

Figure 8. Wave-cres $\ldots$ - s of the symmetric head-on collision of solitary waves with equal amplitude $\sigma_{R}=\sigma_{L}=0.4$ :

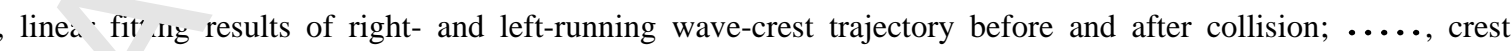
trajectories of our . 'Imerical results; ○ , experimental measurements of Chen \& Yeh [2]. 

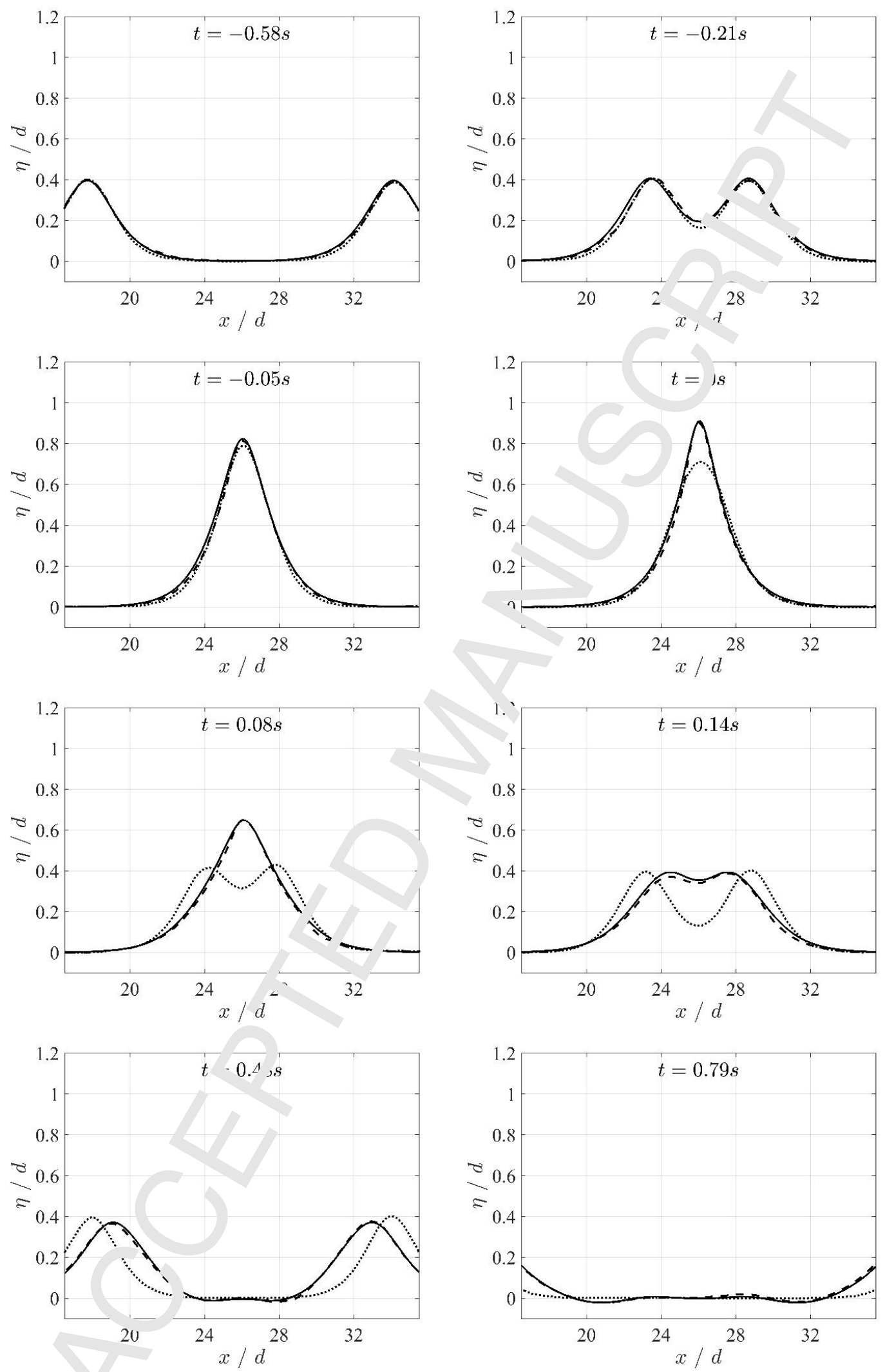

Figure 9. The comparison of free surface profiles during the head-on collision of identical solitary waves with amplitude $\sigma_{R}=\sigma_{L}=0.4:-$, numerical results based on the HPC method; - - - -, experimental results from Chen \& Yeh [2]; $\cdots \cdots$, linear superposition of the counter-propagating KdV solitons. 
Figure. 9 presents the comparison of the numerical, experimental and linear KdV solutions of the free-surface profiles for the head-on collision of solitary waves with amplitudes $\sigma_{R}=\sigma_{L}=0.4$. The moment when the maximum run-up occurs corresponds to $t=0 \mathrm{~s}$ in the experiment of Chen \& Yeh [2]. Satisfactory agreement of our numerical results and the experimental results can be seen in Figure. 9, yet the linear KdV solution fails to predict the colli $>n$ process. On the other hand, we also notice that the wave profile from the experiment slightly differs from our numerival resu " especially for the trailing waves at $t=0.79 \mathrm{~s}$. In fact, the right- and left-running solitary wave in the experimen $\mathrm{w} t \geq$ marginally different in amplitude. The right-running wave was a little higher than the left-running wave after the $\mathrm{co}_{1}{ }^{-}{ }^{-} \mathrm{io}^{\prime}$ in the experiment, which consequently results in the first trailing wave crest behind the right-running wave faintly $h_{c}$ ' her. $L_{c}$ versely, the two waves are the same in amplitude in our numerical study, so that the trailing waves are, corresp $\ldots$. ngly, . mmetric at $t=0.79 \mathrm{~s}$. As mentioned in Chen \& Yeh [2], the phase lag between the linear KdV solution and ey serir inta. measurements was caused by the additional run-up process.

The trailing waves we mentioned above are also manifested in Figure. 10. ' ue to $t$. a asymmetry of solitary waves in the experiment, the phase speed of the right-running wave is slightly larger than ' to minor augment in celerity of the first trailing wave behind the right-runnin, wave. I nus, it can be found, from Figure. 10, that the numerical and experimental trailing wavetrain profiles are in reasc 'ar' $\_$agi ement for the left-running wave, but not for the right-running wave. The first trailing wave trough in the exp imen - - Jpagates slightly faster than that in the numerical calculation. Considering that the trailing waves are minor in an litude, the trailing waves of the present numerical result agree fairly well with the third-order prediction of Su : Mir : [1]. The validation strongly indicates the capability of our numerical model based on the HPC method in successı " $1 \mathrm{v}$ simulating head-on collision of solitary waves.

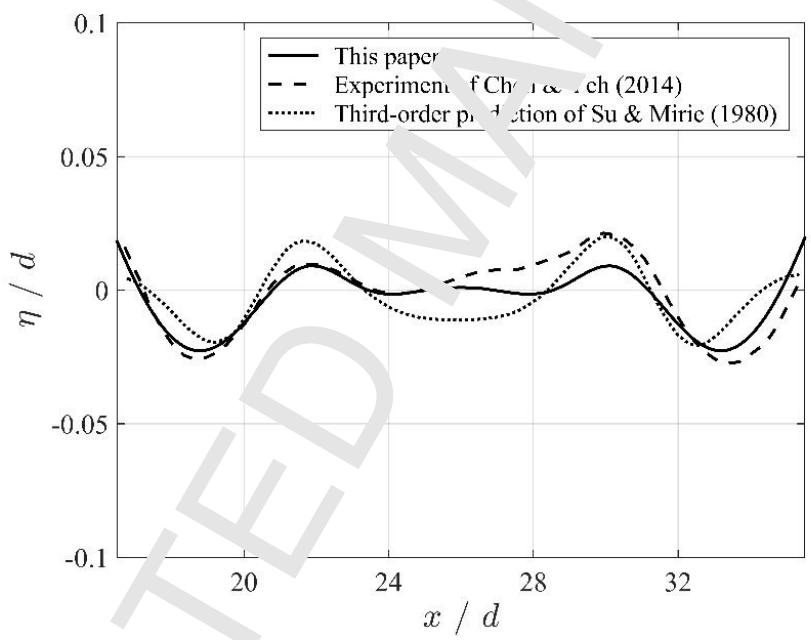

Figure 10. Comparison of dispers; e $\iota_{1}$ iling waves of the present work with experimental result of Chen \& Yeh [2] and the third-order prediction of Su \& M $\therefore$. [ [ $]$ ] at $t=0.96$ s for solitary waves of amplitudes $\sigma_{R}=\sigma_{L}=0.4$.

\section{Analysis of wave-v avr interaction}

4.1 Symmetric and asymmetric : ar $d$-on collision

In this part, we pr sent nu. orical results of head-on collision cases. Four simulation scenarios are considered, which are listed in Table. 3. Th, time $\mathrm{s}^{\dagger}$ p here is $\Delta t=0.02 \mathrm{~s}$.

Table 3. List of numerical simulation scenarios.

\begin{tabular}{|c|c|c|c|c|c|c|}
\hline & Case & $\begin{array}{l}\text { Water depth } \\
\qquad d(\mathrm{~m})\end{array}$ & $\begin{array}{c}\text { Tank length } \\
L(\mathrm{~m})\end{array}$ & $\begin{array}{l}\text { Right-running } \\
\text { wave } \sigma_{R}\end{array}$ & $\begin{array}{l}\text { Left-running } \\
\text { wave } \sigma_{L}\end{array}$ & $\begin{array}{c}\text { Collision center } \\
x / d\end{array}$ \\
\hline Symmetric & 1 & \multirow{4}{*}{1} & \multirow{4}{*}{300} & 0.2 & 0.2 & -40.89 \\
\hline collision & 2 & & & 0.4 & 0.4 & -24.16 \\
\hline \multirow{2}{*}{$\begin{array}{l}\text { Asymmetric } \\
\text { collision }\end{array}$} & 3 & & & 0.2 & 0.4 & -31.16 \\
\hline & 4 & & & 0.3 & 0.4 & -37.56 \\
\hline
\end{tabular}




\section{(a) Symmetric collision}

Figure. 11 shows the spatial-temporal profiles of solitary waves propagating in opposite directions for Case2. As for the phase shifts, Craig et al. [3] proposed that the crest trajectories can be asymptotically expressed as $x=c_{i} t+a_{i}(i=1,2)$ prior to collision and $x=c_{i}^{+} t+a_{i}^{+}(i=1,2)$ after collision, in which $i=1,2$ represent right- nd left-running waves, respectively. Since the phase shift varies with measurement points [23], Craig et al. [3] suggested that $\because 2$ phase shift $\Delta \theta$ can be calculated at the time $t=\tau$ to reduce this variance:

$$
\Delta \theta=\left(a_{i}^{+}-a_{i}\right)+\tau\left(c_{i}^{+}-c_{i}\right),(i=1,2) .
$$

Here $\tau$ is the moment when the maximum run-up occurs during the collision. Figur 12 g. as crest traces of solitary

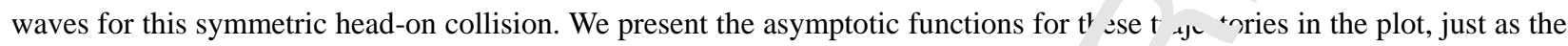
same way as Craig et al. [3] proposed. From the asymptotic functions, we can also $1_{\text {. }}{ }^{2}$ that wave speeds increased after their collision. In this simulation, $\tau / \sqrt{d / g}=426.34$. The phase shifts for righ - and l $\mathrm{ft}$-running wave are calculated by Eq. 13 as $\Delta \theta_{R} / d=0.9459$ and $\Delta \theta_{L} / d=0.9486$, respectively. We have to remark hat thes phase shifts are absolute values representing a retardation of waves after the collision. According to Su \& virie'c [1] third-order prediction, the uniform phase shift for the right- and left-running wave is expressed respectively as:

$$
\left.\begin{array}{l}
\frac{\Delta \theta_{R}}{d}=\left(\frac{\sigma_{L}}{3}\right)^{\frac{1}{2}}\left(1+\frac{1}{8} \sigma_{L}+\frac{3}{4} \sigma_{R}\right) \\
\frac{\Delta \theta_{L}}{d}=-\left(\frac{\sigma_{R}}{3}\right)^{\frac{1}{2}}\left(1+\frac{1}{8} \sigma_{R}+\frac{3}{4} \sigma_{L}{ }_{L}^{\prime \prime}\right)
\end{array}\right\}
$$

However, the phase shifts predicted by Eq. 14 in this case $\mathrm{y}_{1}{ }^{1} \mathrm{~d} . \theta_{R} / d=\Delta \theta_{L} / d=0.49$, which is much lower than the results of our numerical calculation. On the other hand, th. - veri. -ntal measurements of the phase shifts in Chen \& Yeh [2] give 0.98 for the right-running wave and 1.1 for the left-runı. $n g$. ave, which are in reasonable agreement with our present numerical results. The difference between our calcula. ${ }^{\prime} \eta_{s}$ u... Ju \& Mirie's [1] third-order approximation will be further discussed in details in Sec. 4.2.

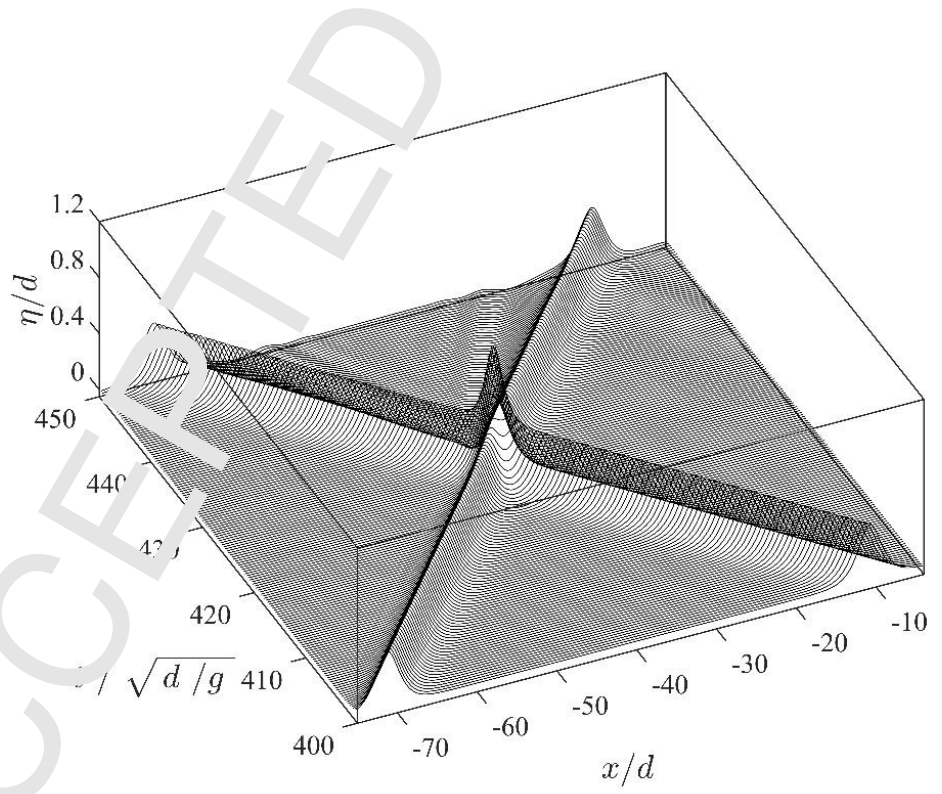

Figure 11. The 'rf e e profiles of head-on collision of solitary waves with an equal wave amplitude $\sigma=A / d=0.4$. 


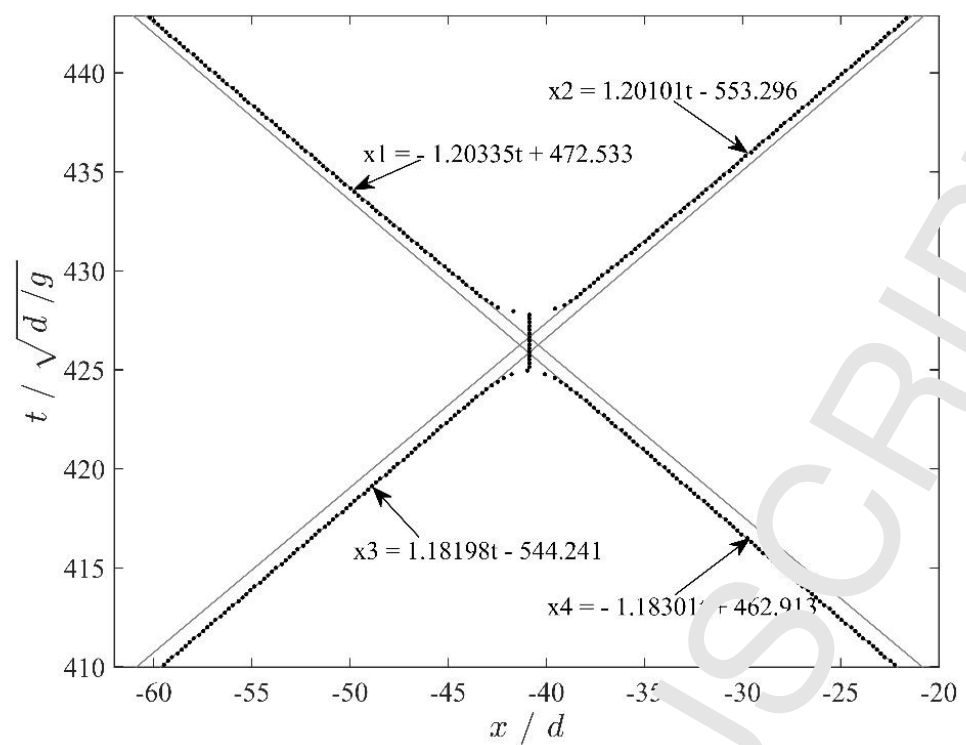

Figure 12. Wave-crest traces of symmetric head-on collision for solita, wav s with an equal amplitude $\sigma=A / d=0.4$ : , linear fitting lines of the right- and left-running wave-crest tra ${ }_{\mathrm{J}}$ - tories before and after the collision; ....., crest trajectories of the present numerical calculations.

(a)

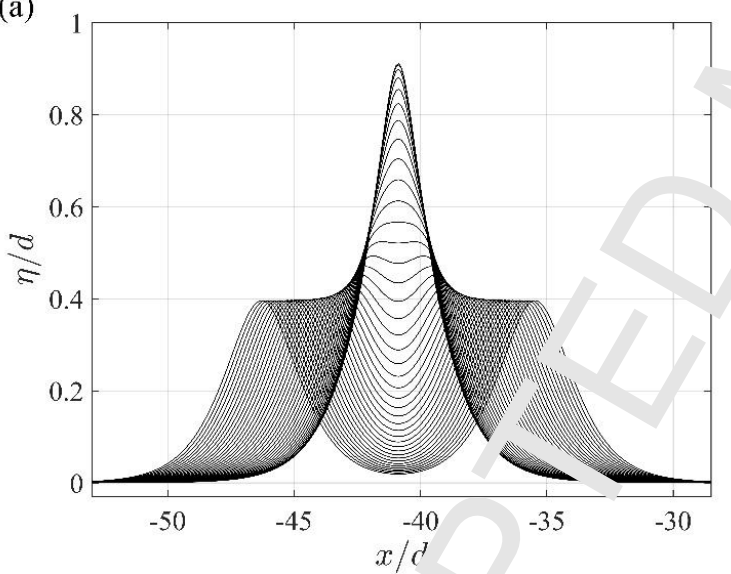

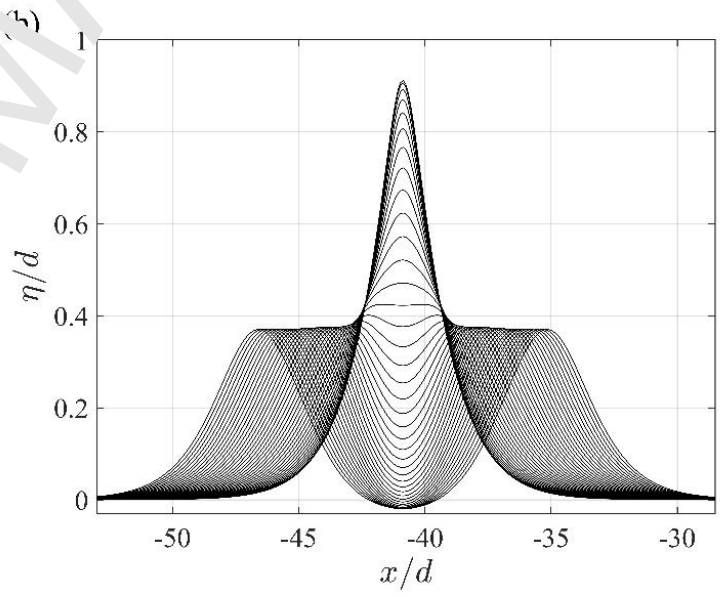

Figure 13. Plots of the free-s' , face pı ciles at different time for waves with amplitudes $\sigma_{R}=\sigma_{L}=0.4$ : (a) before collision for $-10<t / \sqrt{d / g}<0$ and $h)$ a ier $\mathrm{c}^{\prime}$ llision for $0<t / \sqrt{d / g}<10$.

Figure. 13(a) and 7igure. I 3(b) present the collision run-up and run-down process, respectively. We define the time when maximum run-rn oc - s $t=0 \mathrm{~s}$, so the time before the collision is negative $(t<0)$ and the time after the collision is positive $(t>0)$. Fr m Figu. $` 13$, we can clearly find that the maximum run-up during the collision is larger than the sum of two incident amplit. Jes. $T^{1} \approx$ results of the maximum run-up can be found in Table 4. Moreover, the run-up and run-down process of the 1 n collision are not symmetric. In the collision run-up process, the free surface elevation at the collision center gradually is s up with an inflection point of the wave profile at $\eta / d \approx 0.55$, while the inflection point occurs at $\eta / d \approx 0.43$ in the In-down process. In Figure. 13(b), it shows that the amplitude of each wave is slightly attenuated after re-emerging. Furthermore, the wave profile at the collision center is also lower than the quiescent water level between two departing waves. 


\section{(b) Asymmetric collision}

For asymmetric head-on collision, we choose to first generate the higher wave, which travels downstream to be reflected from the tank wall and then collides with the lower wave. In laboratorial experiments, the wave amplitude would attenuate during propagation due to water viscosity and dispersion by reflection from the wall. n our study, despite the absence of water viscosity in potential flow, there is still a dispersion factor causing reduction $n$th produce a reflected solitary wave with a desired amplitude, we need to calibrate the input wavf ... llitude. Take asymmetric collision Case 3 in Table 2 for example, the maximum run-up happens at $\tau / \sqrt{d / g}=40.39$ Figure. 14 displays the collision process between two solitary waves of amplitudes $\sigma_{R}=0.2$ and $\sigma_{L}=0.4$. Fly re. $\mathbf{l}_{\text {- }}$ hows the positions of wave crests prior to and after the collision. The third-order approximation of max . 1lu 1 wavu run-up for asymmetric head-on collision by $\mathrm{Su} \&$ Mirie [1] is

$$
\sigma_{\max }=\sigma_{R}+\sigma_{L}+\frac{\sigma_{R} \sigma_{L}}{2}+\frac{3}{8} \sigma_{R} \sigma_{L}\left(\sigma_{R}+\sigma_{L}\right) \text {. }
$$

In this asymmetric collision, the maximum run-up of the present numerical analys. and $t^{2} \approx$ third-order prediction by Eq.15 are very close to each other, which are summarized in Table 4. In Figure. ${ }^{\mathbf{l}} \boldsymbol{\jmath}$, the ${ }^{-}$maller wave is gradually swallowed by the larger one until only one wave crest is formed in the run-up process. ' n' . . e th symmetric collision, the single crest moves with a changing speed instead of standing at a fixed point, which ca. also u seen in Figure. 15 in the collision area (marked as + ). In the run-down process, both the two waves do not re-gain th ir initial amplitudes and the smaller wave reemerges from the single crest.

Table 4. Non-dimensional results of symmeı. and asymmetric head-on collision.

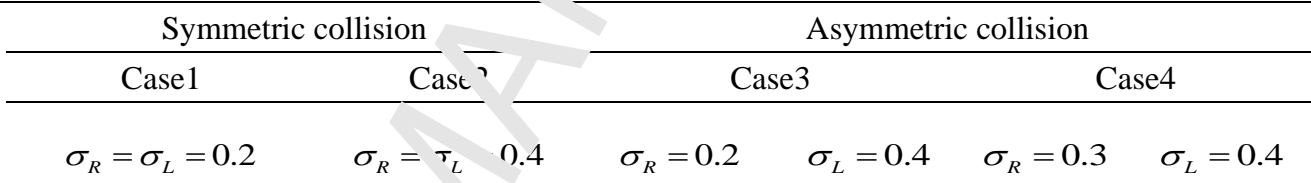

\begin{tabular}{|c|c|c|c|c|c|c|c|c|}
\hline $\begin{array}{l}\text { Third-order approximation } \\
\text { of the maximum run-up }\end{array}$ & \multicolumn{2}{|c|}{0.4260} & \multicolumn{2}{|c|}{ ย. ว 80} & \multicolumn{2}{|c|}{0.6580} & \multicolumn{2}{|c|}{0.7915} \\
\hline Numerical maximum run-up & \multicolumn{2}{|c|}{0.4243} & & 18 & \multicolumn{2}{|c|}{0.6486} & \multicolumn{2}{|c|}{0.7815} \\
\hline Amplitude after collision & 0.1889 & $\overline{0.18}=$ & $\overline{0.3} \overline{86}$ & 0.3790 & 0.1939 & 0.3857 & 0.2847 & 0.3841 \\
\hline Reduction ratio of amplitude & $5.56 \%$ & $510 \%$ & $-3 \overline{5 \%}$ & $5.25 \%$ & $3.05 \%$ & $3.57 \%$ & $1.53 \%$ & $5.30 \%$ \\
\hline
\end{tabular}

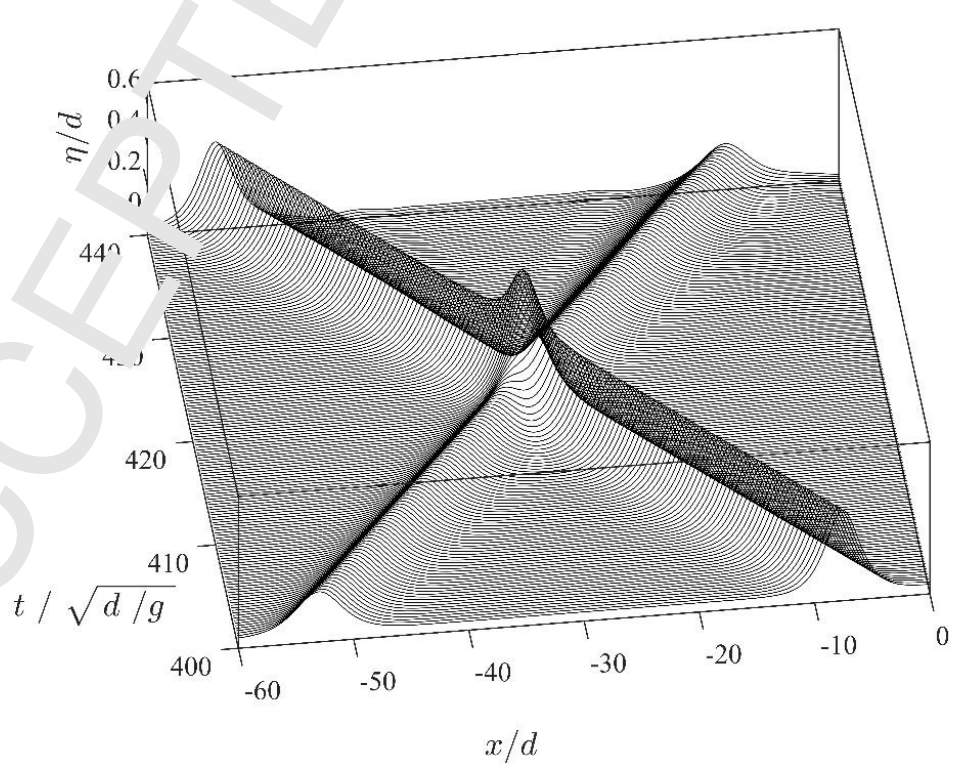

Figure 14. The surface profiles of the head-on collision of solitary waves with wave amplitudes $\sigma_{R}=0.2$ and $\sigma_{L}=0.4$. 


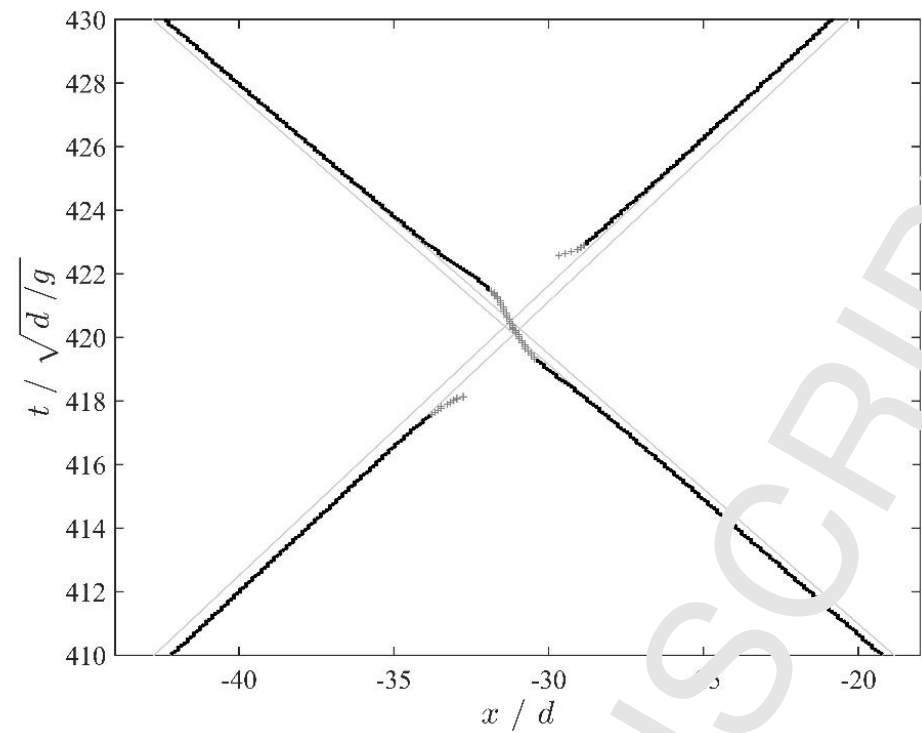

Figure 15. Wave-crest traces of the asymmetric head-on collision for solitary waves with wave amplitudes $\sigma_{R}=0.2$ and $\sigma_{L}=0.4$ : , linear-fitting lines of right- and left-running wav -rest iajectories prior to and after the collision;

(a)

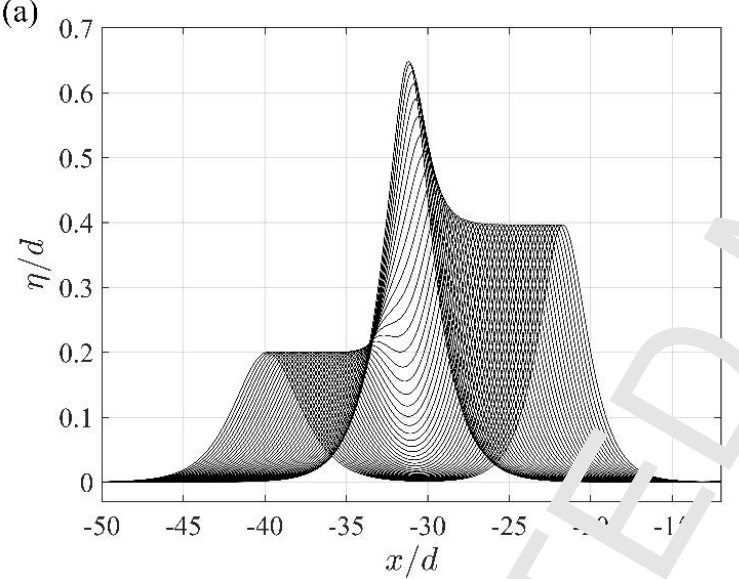

(b)

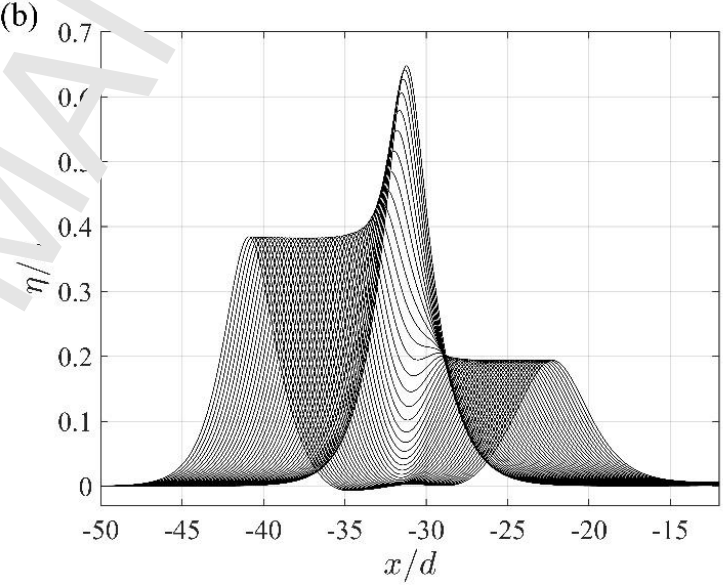

Figure 16. Plots of the temporal variacion os he free surface profile for wave amplitudes $\sigma_{R}=0.2$ and $\sigma_{L}=0.4$ : (a) before the collision for $-8.3<t / \sqrt{d /} g^{\prime}<$; (b) after the collision for $0<t / \sqrt{d / g}<8.3$.

\subsection{Investigation of phase $s^{\prime}$ ifts for :-e head-on collision}

As mentioned in Sec. / 1, C ten $\delta$, Yeh's [2] experimental results of the phase shifts do not agree with the third-order prediction by $\mathrm{Su} \&$ Mirie [11. $1_{1}$. ${ }^{\prime}$ screpancy was also noticed by Craig et al. [3] in their fully nonlinear numerical study. In this part, we will acc unt for ^ e reason why the prediction by Eq. 14 underestimates the phase shifts.

For the symmetric ollisior, the free surface profile at the collision center is gradually elevated as the two solitary waves approach er $n$ other in the run-up process. At the same time, the amplitude of each wave grows until the wave amplitude is equa to the fr e surface elevation at the collision center. The process corresponds to the appearance of the inflection of the wave,$\ldots . . .1$ in Figure. 13. During this process, there is a moment when the wave speed is close to infinity until a single ci st a pruis at the collision center. This observation can be referred to the theoretical analysis of Cooker et al. [26]. It can be fou 4 from Figure. 17 that some crest trajectories (symbols ' + ') close to the collision center deviate from the fitting lines due to the dramatic growth of wave speed in a short period. The inclusion of these crest trajectories close to the collision center to get the linear fitting lines will affect the calculations of the phase shifts by Eq. 13. In this case, these trajectories close to the collision center are reasonably discarded while obtaining the linear fitting lines by a least-squared method. In the following texts, for simplicity we will use 'deviated data' and 'fitting data' to stand for the crest trajectory 
data close to the collision center and remnant fitting data, respectively.

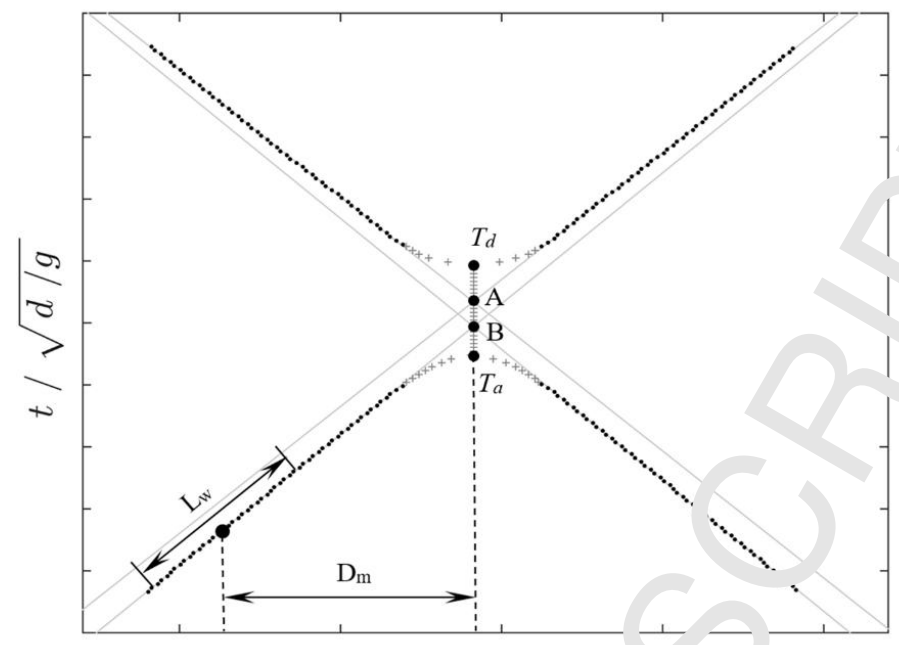

$x / d$

Figure 17. Wave-crest traces of symmetric head-on collision: , line. - fitting lines of crest trajectories; - and + , the present numerical results.

To calculate the phase shifts by Eq. 13, the main task is to $s_{\mathrm{r}}$ ' $1 r y$ the linear fitting functions based on the 'fitting data' prior to and after the head-on collision. We find that the fitting ' inction varies with both the distance $D_{\mathrm{m}}$ from mearsurement point to the collision center and the length of $\downarrow$ ta /lluow $L_{\mathrm{w}}$, which are demonstrated in Figure. 17. The measurement point is fixed at the center of each data wir ' 1 w. 1. a following model of window length $L_{\mathrm{w}}$ is conducted in specifying the linear fitting functions:

$$
L_{\mathrm{w}}=\left\{\begin{array}{l}
{[5,10], \text { if } D_{\mathrm{m}}^{-11,1 \mathrm{v}_{\mathrm{J}}}} \\
(10,20], \text { if } D_{\mathrm{m}} \in(1 \mathrm{v}, 20] \\
\left(20,40, \text { if } L_{\mathrm{L}} \in(20,40]\right. \\
\left(40,6 \mathrm{u}_{\lrcorner}, \text {if } D \in(40,+\infty]\right.
\end{array} .\right.
$$

The slope of wave-crest trace changes drar atically close to the collision center. Therefore, the data window should be shorter. However, considering there still $\mathrm{e}_{\mathrm{\lambda}}$. nur erical errors when recording the crest trajectories, the window length should not be set too small to avoid d; 'ortion of the fitting lines. For the measurement point closer to the collision center, the calculation of the phase shifts shows that ' $\mathbf{~} \mathbf{1 6}$ can perform well in getting fitting lines based on 'fitting data'.

(a)

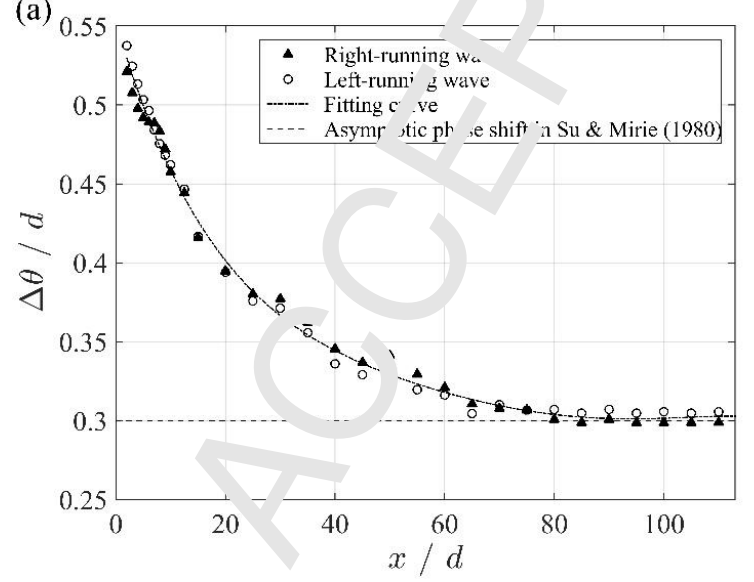

(b)

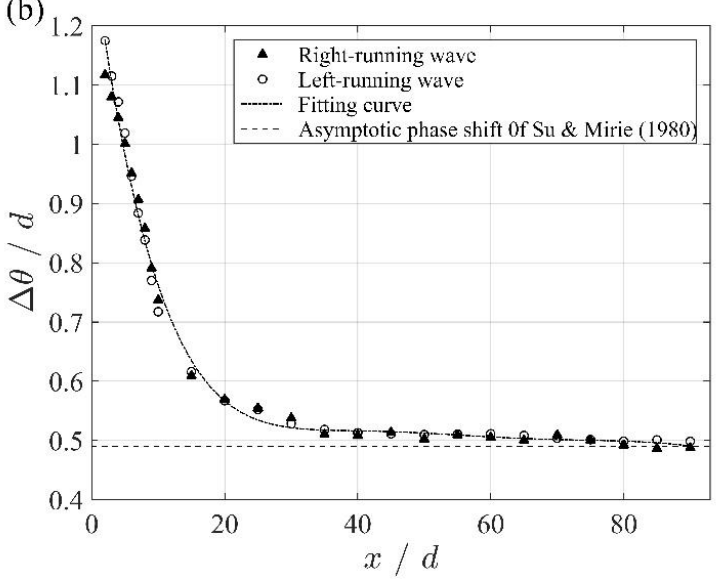

Figure 18. Phase shifts versus the measurement distance from the collision center for symmetric head-on collision of solitary waves: (a) $\sigma_{R}=\sigma_{L}=0.2$ and (b) $\sigma_{R}=\sigma_{L}=0.4$. The asymptotic value for $\sigma_{R}=\sigma_{L}=0.2$ and $\sigma_{R}=\sigma_{L}=0.4$ is $\Delta \theta / d=0.3$ and $\Delta \theta / d=0.49$, respectively. 
Fenton \& Rienecker [23] pointed out that the measurement of phase shifts is sensitive to the distance from the collision center. They found that their numerical results of the phase shifts near to the collision center are in consistent with Su \& Mirie's [1] another third-order phase shift limit, namely

$$
\left.\begin{array}{l}
\frac{\Delta \theta_{R}}{d}=\left(\frac{\sigma_{L}}{3}\right)^{\frac{1}{2}}\left(1+\frac{1}{8} \sigma_{L}+\frac{23}{4} \sigma_{R}\right) \\
\frac{\Delta \theta_{L}}{d}=-\left(\frac{\sigma_{R}}{3}\right)^{\frac{1}{2}}\left(1+\frac{1}{8} \sigma_{R}+\frac{23}{4} \sigma_{L}\right)
\end{array}\right\},
$$

which is called immediate phase shift. The immediate phase shift by Eq. 17 for $c_{R}=\iota_{L}=0.2$ and $\sigma_{R}=\sigma_{L}=0.4$ is $\Delta \theta / d=0.56$ and $\Delta \theta / d=1.22$, respctively. Excellent agreement can be observed from . . "Ire. 18, between our numerical results near to the collision center and the prediction by Eq. 17.

For $\sigma_{R}=\sigma_{L}=0.4$, Chen \& Yeh [2] dealt with their experimental data, actu. 'ly, usin, the same window model as we proposed in Eq. 16 and obtained $\Delta \theta_{R} / d=0.98$ and $\Delta \theta_{L} / d=1.1$. The 'deviater' uata' were also discarded in their analysis. We can translate their 'fitting data' into our window model: $D_{\mathrm{m}} \approx 5$ and $L_{\mathrm{w}} \approx 6.5$ Or' calc lation yields $\Delta \theta_{R} / d=1.0$ and $\Delta \theta_{L} / d=$ 1.01, which are in good agreement with the experimental results. On top $f$ this . .other satisfactory discovery is that the calculated phase shifts are asymptotic to the prediction by Eq. 14 if the measu ement point is far away from the collision center. The approximation of the phase shifts by Eq. 14 is named as the . ifor , phase shift when two departing waves are far enough away from each other. Chen \& Yeh [2] thought their calcula. 'n of the phase shift based on the 'fitting data' is larger than the asymptotic value of Eq. 14 mainly resulting fre ' ne solitary wave tilting backward with respect to the direction of its propagation after the collision. In fact, the 'fitting a -' used by Chen \& Yeh [2] are very close to the collision center. The result they calculated corresponds, actu ly. co we immediate phase shift, but they compared their immediate phase shift with the asymptotic value of the 1 : form, hase shift by Eq. 14. This is the main reason why they mistakenly concluded that the third-order prediction (Eq. 14, of Su \& Mirie [1] could not predict their experimental measurements of the phase shift.

(a)

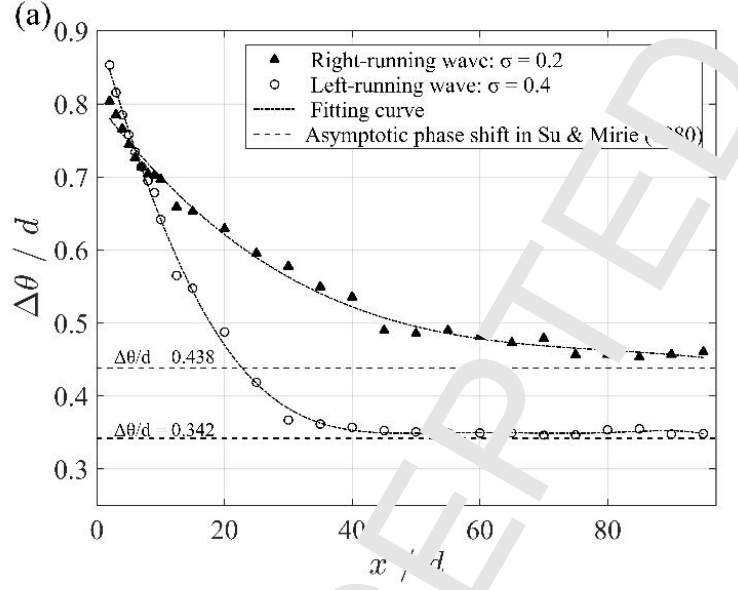

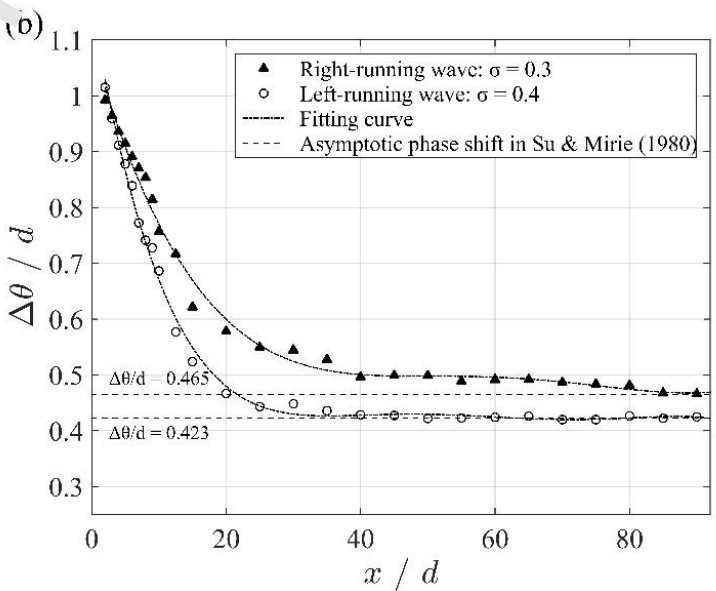

Figure 19. Phase shift versus he measurement distance from the collision center for asymmetric head-on collision of solitary waves: (a) $\sigma_{R}=\iota ?, \sigma_{L}=J .4$ and (b) $\sigma_{R}=0.3, \sigma_{L}=0.4$.

Figure. 19 pi vides pl ase shifts versus the measurement distance from the collision center for asymmetric head-on collision. Both the in..... ate phase shifts and the uniform phase shifts of our calculations are in good agreement with the third-order asyı nto ${ }^{+} \mathrm{c}$ ı sults. We can notice that the retardation of the smaller solitary wave is more severe than the higher wave considering, e uniform phase shift, which can also be seen from Figure. 15.

As we have mentioned above, the measurement of the phase shifts strongly depends on the distance from the collision center because of the change of wave speed after the collision. Fenton \& Rienecker [23] found that even though the spatial phase shift changes with measurent positions, the phase lag in time $\Delta T$ remains unchanged at the collision center, which is equivalent to the length of $\overline{A B}$ segment in Figure. 17 for symmetric head-on collision. However, it is still difficult to 
evaluate $\Delta T$ in practice because the location of point A and B in Figure. 17 depends on the linear fitting lines. As we have discussed, the determination of the fitting functions is associated with $D_{\mathrm{m}}$ and $L_{\mathrm{w}}$. Under this circumstance, Cooker et al. [26] proposed to use the wall residence time $T_{r}=T_{d}-T_{a}$, alternatively, as the definition of phase lag in time. $T_{a}$ is the attachment time at which the incoming wave arrives at the vertical wall and $T_{d}$ is the detachment $t^{;}$ie at which the reflected wave leaves the wall. The equivalent attachment and detachment time for symmetric head-on colısion as defined in Figure. 17. The wall residence time given by Cooker et al. [26] is

$$
\frac{T_{r}}{\sqrt{d / g}}=\frac{4 \kappa_{0}}{\sqrt{3}}\left(\sigma^{-1 / 2}+\frac{1}{8} \sigma^{1 / 2}\right),
$$

where $\kappa_{0}=\tanh ^{-1}(1 / \sqrt{3})$.

Figure. 20 records the maximum amplitude versus time for symmetric $\mathrm{h}$ d dd-on c llision. Figure. 20(a) shows the comparison of the present results with the numerical results of Cooker et l. [26] or $\sigma_{R}=\sigma_{L}=0.2$ with excellent agreement. Figure. 20(b) gives the present numerical result, experimental re utt of (nen \& Yeh [2] and numerical result of Craig et al. [3] for $\sigma_{R}=\sigma_{L}=0.4$. Our numerical result of maximum wave mr' .ude agrees well with Cooker et al.'s [26] numerical result, while the experimental result of the maximum run- $u_{1}$ is $_{s} s^{-11} \mathrm{f}$ than our numerical result. The wall residence time of our numerical results and the solutions by Eq.18 for diffe nt cases are summarized in Table 5. For asymmetric collision, Eq.18 is not applicable.

Table 5. The wall residence time for symmetric collisions . '1 rully interacting time for asymmetric collisions.

\begin{tabular}{|c|c|c|c|c|}
\hline $\begin{array}{l}\text { Wall residence time or } \\
\text { fully interacting time }\end{array}$ & $\begin{array}{l}\text { Type of } \\
\text { collision }\end{array}$ & an. & $\begin{array}{c}\text { Present } \\
\text { numerical result }\end{array}$ & $\begin{array}{l}\text { Theoretical result } \\
\text { (Eq.18) }\end{array}$ \\
\hline$T_{r}$ & \multirow{2}{*}{$\begin{array}{l}\text { Symmetric } \\
\text { collision }\end{array}$} & $o_{\Lambda} \quad \tau_{L}=7.2$ & 3.633 & 3.672 \\
\hline$\sqrt{d / g}$ & & $-=c=0.4$ & 2.944 & 2.970 \\
\hline$T_{f}$ & \multirow{2}{*}{$\begin{array}{c}\text { Asymmetric } \\
\text { collision }\end{array}$} & $\sigma_{R}=0 . .4 \mid \sigma_{L}=0.4$ & 4.323 & NA \\
\hline$\sqrt{d / g}$ & & $\sigma_{R}=0.3 \mid \sigma_{L}=0.4$ & 3.630 & NA \\
\hline
\end{tabular}

(a)

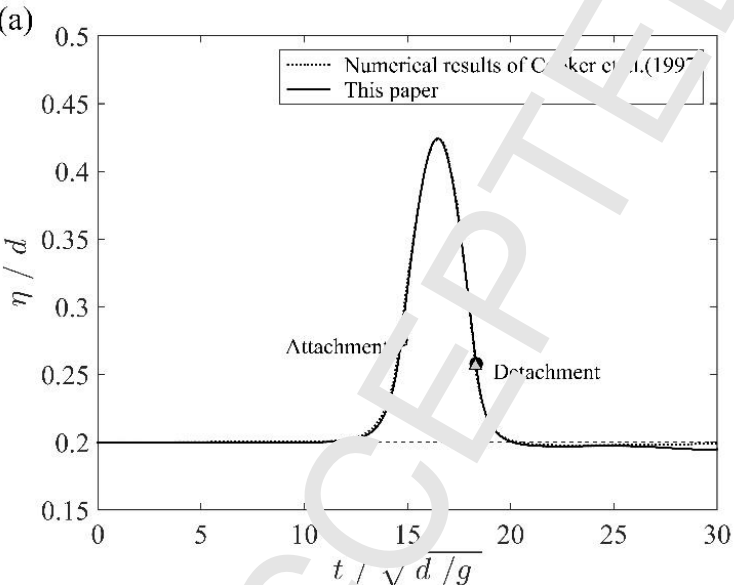

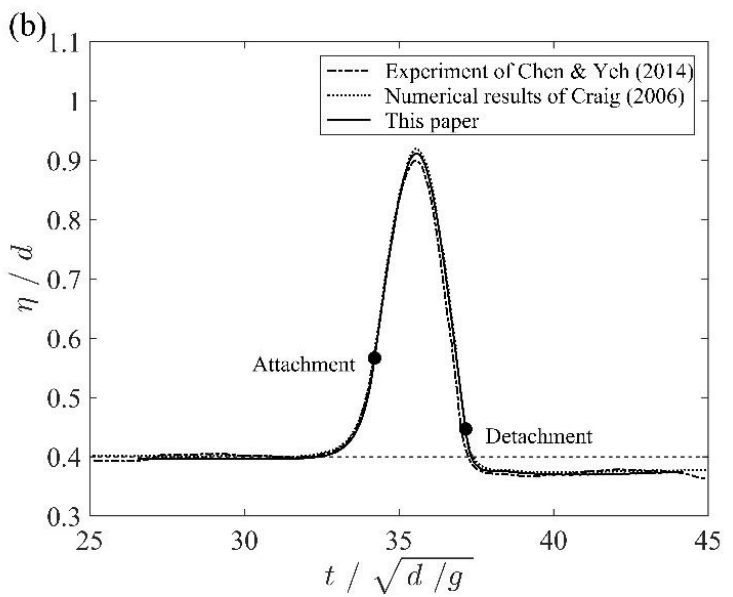

Figure 20. Tempo 'l variat; in of the maximum amplitude for symmetric head-on collision. (a) The comparison of the our numerical results wilı ... numerical results of Cooker et al. [26], here $\sigma_{R}=\sigma_{L}=0.2$. (b) The comparison of the our results with th $\mathrm{nv}$ nerical results of Craig et al. [3] and the experimental measurements of Chen \& Yeh [2], here $\sigma_{R}=\sigma_{L}=0.4$. A. xchment and detachment points are indicated: $\Delta$ from Cooker et al. [26] and • from the present study. 
(a)

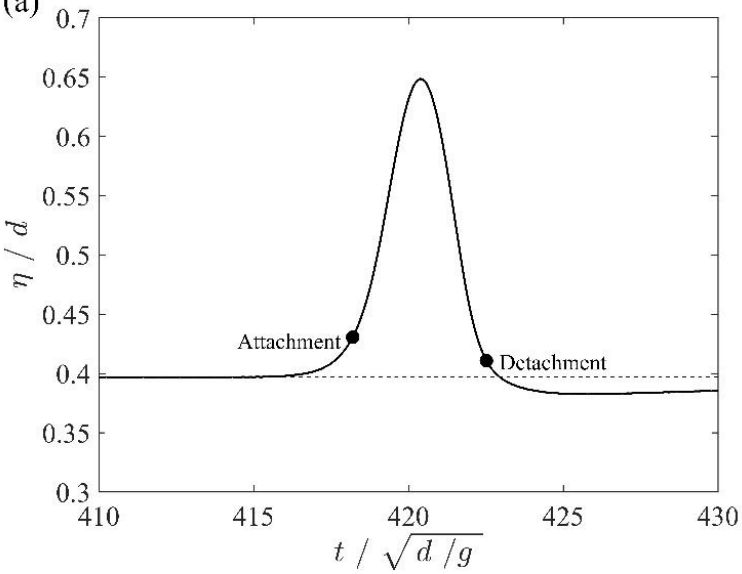

(b)

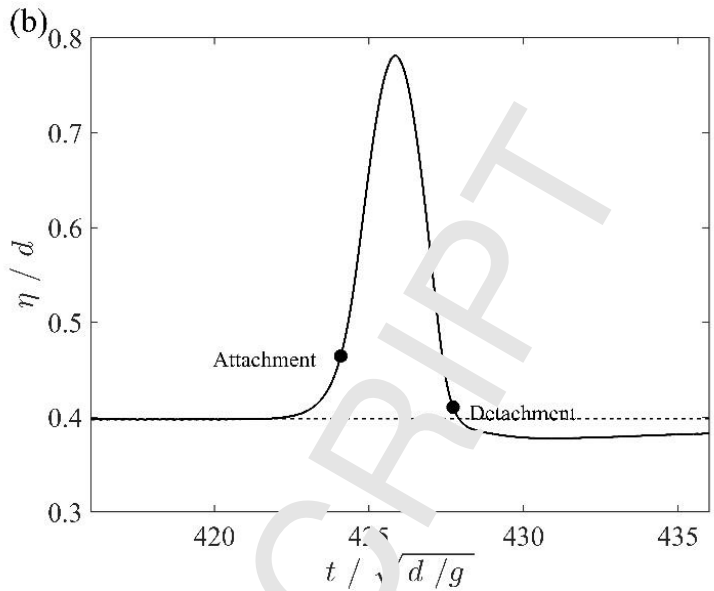

Figure 21. Temporal variation of the maximum amplitude for asymmetric atd-on cullision. (a) $\sigma_{R}=0.2$, $\sigma_{L}=0.4$. (b) $\sigma_{R}=0.3, \sigma_{L}=0.4$. Attachment and detachment point are indicated.

Figure. 21 shows the maximum amplitude versus time of the higher $s$ litary wave for both the two asymmetric head-on collision cases, i.e. $\sigma_{L}=0.4$. During the collision, we can alv. vs re ord the crest trajectory of the higher wave because the higher wave gains height without losing its crest by swallu ing the smaller one. On the other hand, there is a dramatic leap of the crest elevation for the smaller solitary wave. ven it is swallowed, shown in Figure. 16.

Although the attachment and detachment point are proposed in is a study on the reflection of a solitary wave from a vertical wall, we can still identify them in asymmetric head- $\eta \mathrm{cr}$.... $\mathrm{j}$. In fact, the attachment and detachment point in symmetric or asymmetric head-on collision are the critic ${ }^{1}$ time $\cdot$ divide the whole collision process into two parts. There are two crests before the attachment and after the detachme t, hile there is only a single crest between the attachment and detachment time. So we can define the 'wall residener ':me' in 'vall reflection of a solitary wave as general fully interacting time $T_{f}$, namely

$$
T_{f}=T_{d}-T,
$$

which can be applied in both symmetric and asymme ric head-on collision. The numerical results of two asymmetric collision cases are summarized in Table 5. No re. ' $9 r c^{\prime}$ ers have investigated the analytical expressions of the attachment and detachment time for the asymmetric c llisi' $n$ according to authors' knowledge, which requires further research in the future.

\subsection{Overtaking collision}

Overtaking collision of two s' 1ta.' waves is considered as a 'strong' wave-wave interaction, which means that it takes a long duration for a larger solita. w ve to catch up with, interact with, overtake and separate from the smaller one. There is a slight modification of wa' $\_$amplıь ' 2 and velocity after the collision, which has been discussed by Craig et al. [3] and

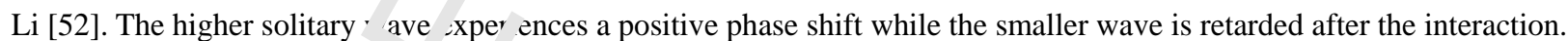

In order to determine the in ner cal phase shifts of solitary waves after the overtaking collision, we take two locations $X_{1}$ and $X_{2}\left(X_{2} \geq X_{1}\right)$.' ' hen $\mathrm{p}_{\mathrm{c}}$ 'sing these locations, the two solitary waves are clearly separated. The collision domain is between these two locatı $\mathrm{ns}$. The phase shifts can be evaluated according to $\mathrm{Li}[52]$ as

$$
\hat{s}_{i}=L_{0}-c_{i} \Delta t_{i},(i=1,2),
$$

where $L_{0}=X_{2}-X_{1}$ nd $c_{i}(=1,2)$ is the ninth-order phase speed according to Fenton [19]. $\Delta t_{i}(i=1,2)$ is the time interval of a wave crest $\mathrm{p} ;$ - these two locations.

Li [52] gave ${ }^{4} \mathrm{e}$ theoretical phase shifts for the two solitary waves in the form of

$$
\left.\begin{array}{l}
s_{1}=\frac{1}{\tilde{\mu}_{1}} \ln \frac{\left(\tilde{\mu}_{2}-\tilde{\mu}_{1}\right)^{2}}{\left(\tilde{\mu}_{2}+\tilde{\mu}_{1}\right)^{2}} \\
s_{2}=\frac{1}{\tilde{\mu}_{2}} \ln \frac{\left(\tilde{\mu}_{2}-\tilde{\mu}_{1}\right)^{2}}{\left(\tilde{\mu}_{2}+\tilde{\mu}_{1}\right)^{2}}
\end{array}\right\},
$$


1 where $\tilde{\mu}_{1}=\sqrt{3 \sigma_{1}}, \quad \tilde{\mu}_{2}=-\sqrt{3 \sigma_{2}}, \quad \sigma_{1}=A_{1} / d$ and $\sigma_{2}=A_{2} / d$ are dimensionless wave amplitude of the higher solitory wave and the smaller one, respectively. $d$ is the calm water depth. Since $s_{1}>0$ and $s_{2}<0$, the higher wave is shifted forward and the smaller wave shifts backward after the interaction.

Lax [28] has categorized the overtaking collision of two solitary waves based on the raıo bet. an the two initial amplitudes $\sigma_{1} / \sigma_{2}\left(\sigma_{1}>\sigma_{2}\right)$ : (a) $1<\frac{\sigma_{1}}{\sigma_{2}}<\frac{3+\sqrt{5}}{2}$, (b) $\frac{3+\sqrt{5}}{2}<\frac{\sigma_{1}}{\sigma_{2}}<3$, (c) $\frac{\sigma_{1}}{\sigma_{2}}>3$. Howeve. Cra g et al. [3] found that the category given by Lax [28] seemed unable to accurately predict larger amplitude $r_{\text {. }}$ cases, and proposed a revised criterion based on their experimental and numerical results, namely

$$
\begin{gathered}
1<\frac{\sigma_{1}}{\sigma_{2}} \leq 2.941, \text { category (a); } \\
2.941<\frac{\sigma_{1}}{\sigma_{2}} \leq 3.536, \text { category (b); } \\
\frac{\sigma_{1}}{\sigma_{2}}>3.536, \text { category (c) } .
\end{gathered}
$$

In the present study, selected overtaking collision cases will be simulate and the results will be validated with the exsiting numerical and experimental results. The present numerical resuı will sso be used to check the applicability of the categories proposed in Lax [28] and Craig et al. [3].

(a)

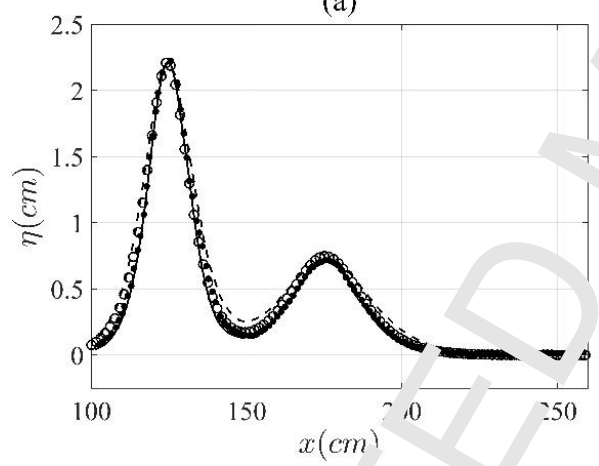

(c)

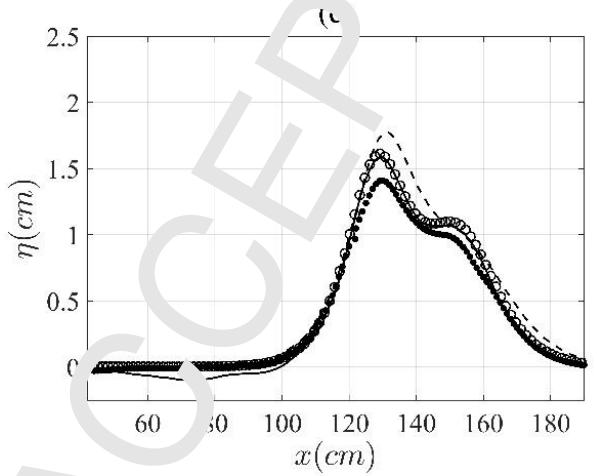

(b)

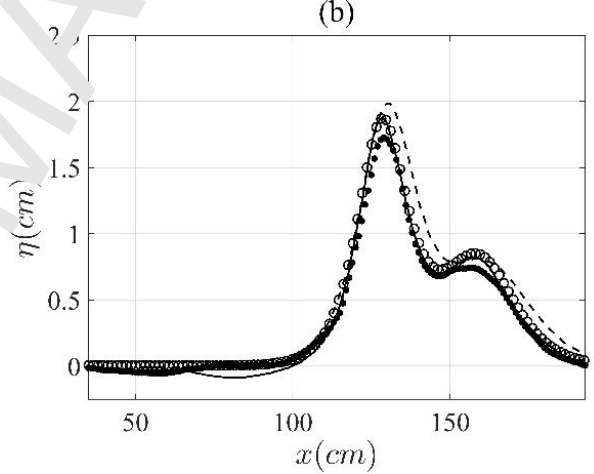

(d)

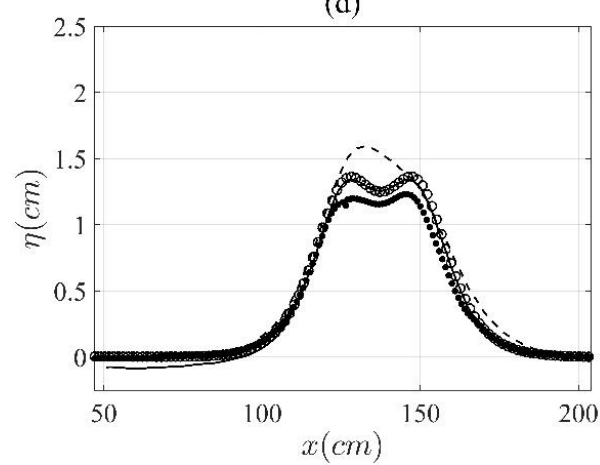


(e)

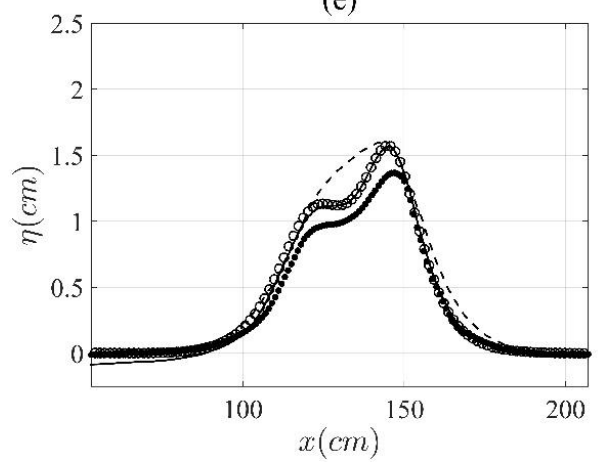

(g)

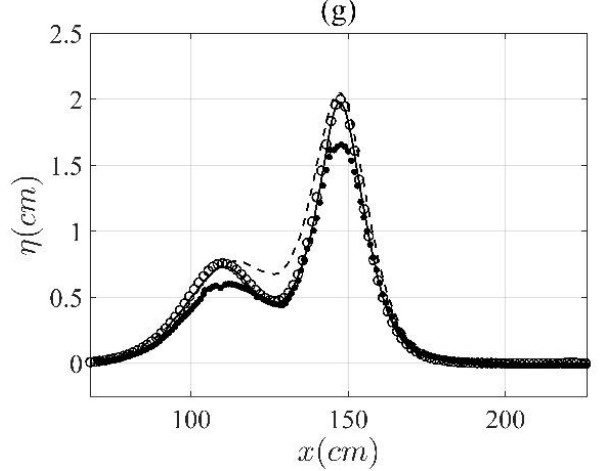

(f)

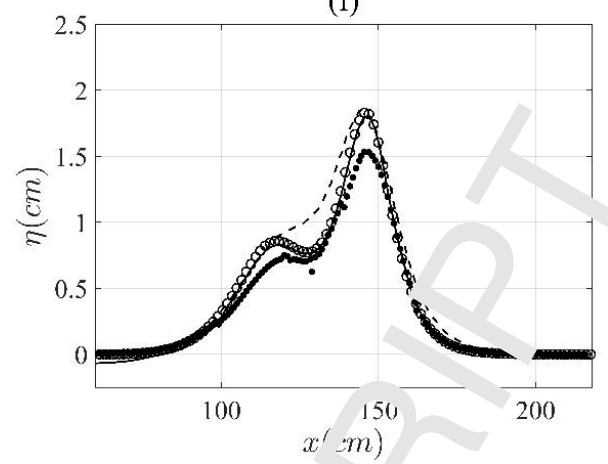

(h)

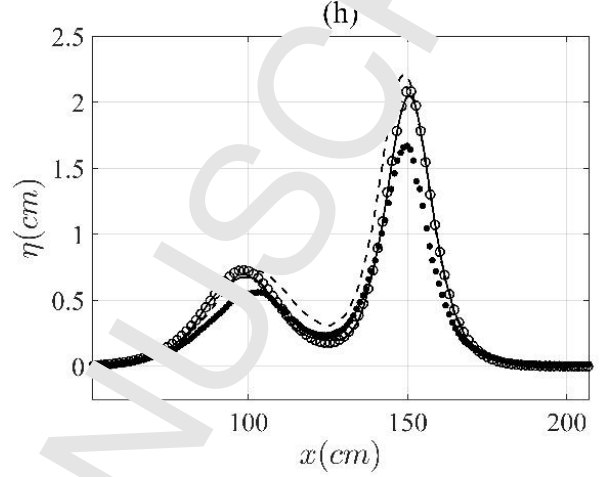

Figure 22. Overtaking collision of solitary waves with amplitudes $A_{1}=? .95 \mathrm{~cm}$ and $A_{2}=0.730 \mathrm{~cm}$ at (a) $t=2.90304 \mathrm{~s}$, (b) $t=$ $5.50196 \mathrm{~s}$, (c) $t=6.40517 \mathrm{~s}$, (d) $t=7.05025 \mathrm{~s}$, (e) $t=7.60014 \mathrm{~s}, \quad$ ) $t=0.00024 \mathrm{~s}$, (g) $t=9.50478 \mathrm{~s}$, (h) $t=11.30191 \mathrm{~s}$. Here, - numerical result of Craig et al. [3]; - - - - , lin $\neg \mathrm{Kd}$, solution; $\cdots .$. , experimental result of Craig et al. [3]; O, our present result.

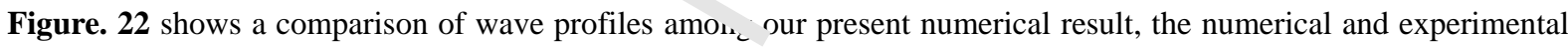
result of Craig et al. [3] and the KdV two-soliton "'tion at different time. The quiescent water depth is $d=5.0 \mathrm{~cm}$. It can be observed that the present numerical result ar ee well ith the numerical result of Craig et al. [3]. The overtaking process takes a long distance so that we can notice th $>$ re $1 \mathrm{~s}$. ' $\mathrm{o}^{1}$ vious attenuation in the wave amplitudes due to the water viscosity in Craig et al.'s [3] experiment. Despite th war - amplitudes decrease with the distance, the wave profile still has the same form as numerical results at $t=7.05025$ s $\left(\mathbf{F}_{\boldsymbol{r}_{c}}\right.$ re. $\left.?(\mathbf{d})\right)$, while the linear $\mathrm{KdV}$ solution has already been unable to provide convincible prediction.

Figure. 23 demonstrates a higier soı $\wedge^{-r v}$ wave of $\sigma_{1}=0.4$ catching up a smaller wave with amplitudes $\sigma_{2}=0.3$, $0.133,0.113$ and 0.1 , respectively sin $\geqslant$ the wave velocity is dependent on wave amplitude, the duration of the overtaking process is different from case to - ce the length of the wave tank is much longer compared with head-on collision. In this study, the tank length has be $\mathrm{n}$ adjusı ${ }^{1}$ to avoid wave reflection from the downsteam tank wall before the overtaking process is completed. The $\mathrm{w}$ ter $r^{\prime} \mathrm{sth} ; d=1 \mathrm{~m}$ in our simulations.

It is difficult to tell from $\mathbf{F}_{\mathbf{3}} \cdot \mathbf{w} . \mathbf{2 3}(\mathbf{a})$ whether the collision have happened or not, but we can identify the interaction from Figure. 23(b). Dv ing the ollision, the amplitude of the larger solitary wave monotonically decreases as the smaller wave rises up until the $\mathrm{L}$. air am ${ }^{r}$,itudes become identical at a critical time. After the critical time, the process reverses till two wave crests arf well senarated. Specifically, there are two distinguishable crests during the collision process. The whole interaction betwee solitary vaves of $\sigma_{1}=0.4$ and $\sigma_{2}=0.3$ takes a very long CPU time as the relative velocity of the two waves is too small. A... . ne collision, the higher solitary wave shifts forward while the smaller one shifts backward. Our numerical resu 's to . ....s case with ratio $\sigma_{1} / \sigma_{2}=1.333$ matches with both the KdV category (a) by Lax [28] and the category (a) by $\mathrm{Cr}$, o et al. [3]. 
1

(a)

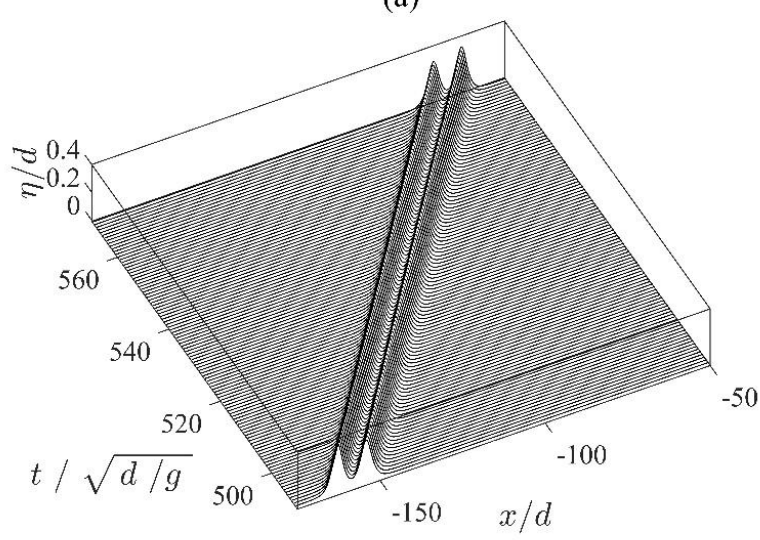

4

7 (c)

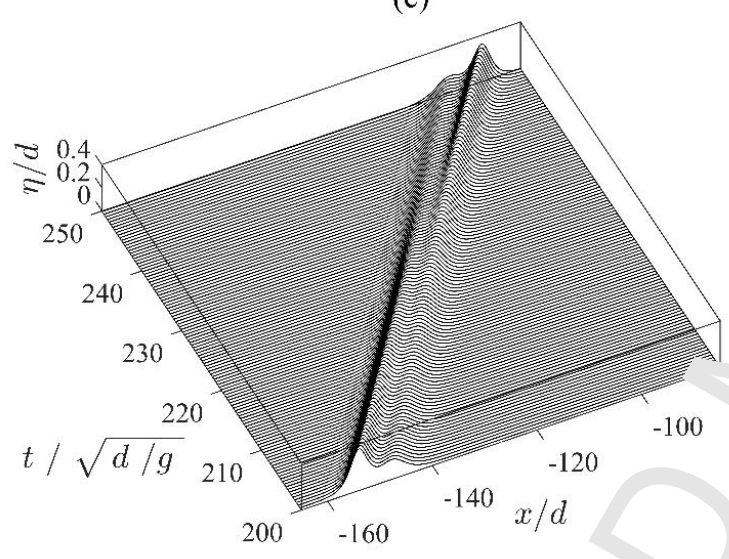

(c)

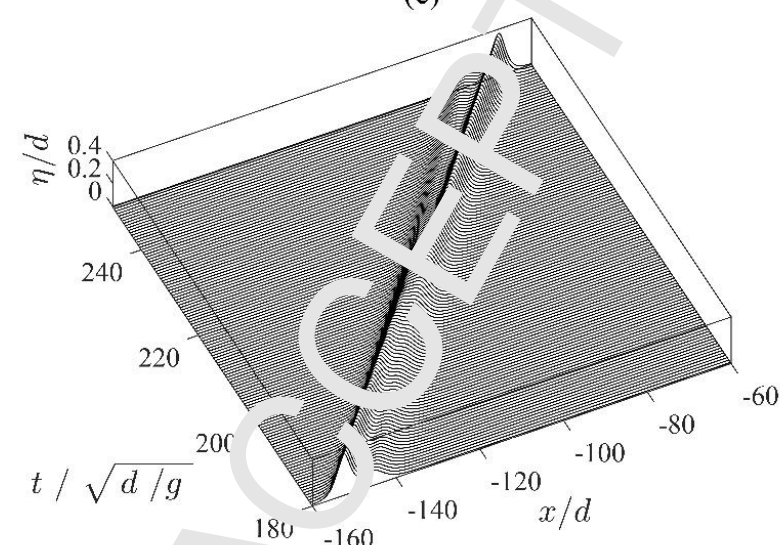

(b)

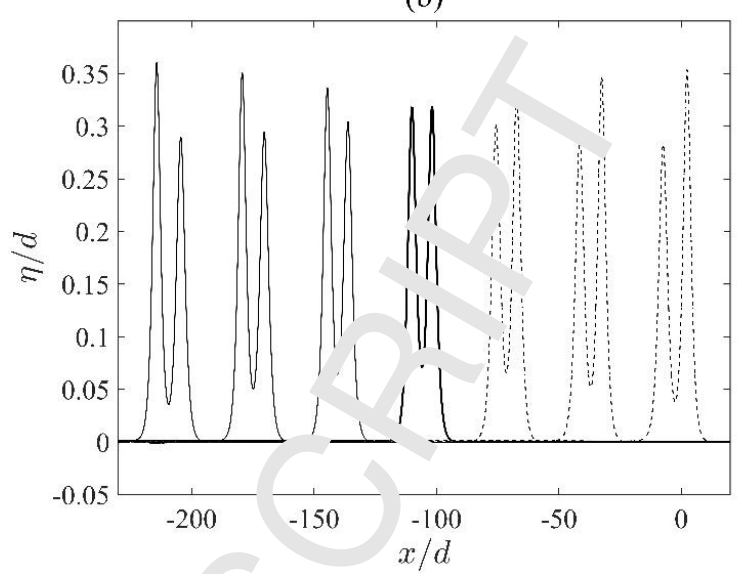

(d)

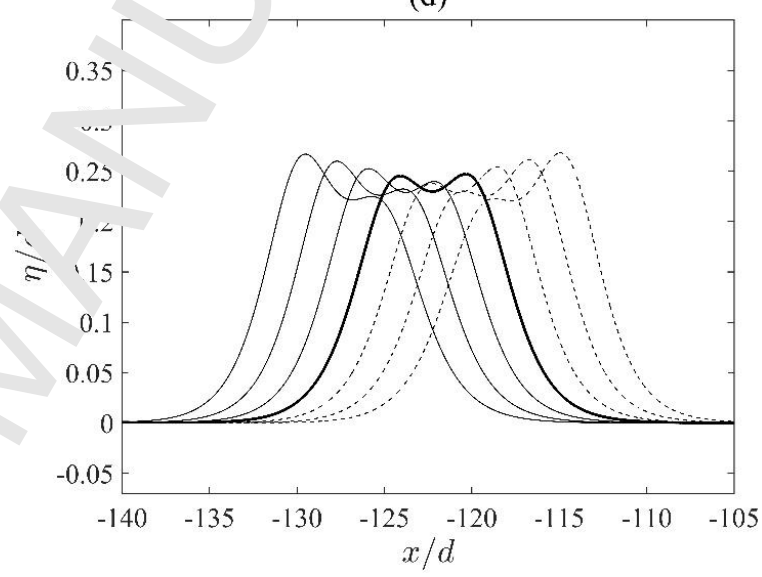

(f)

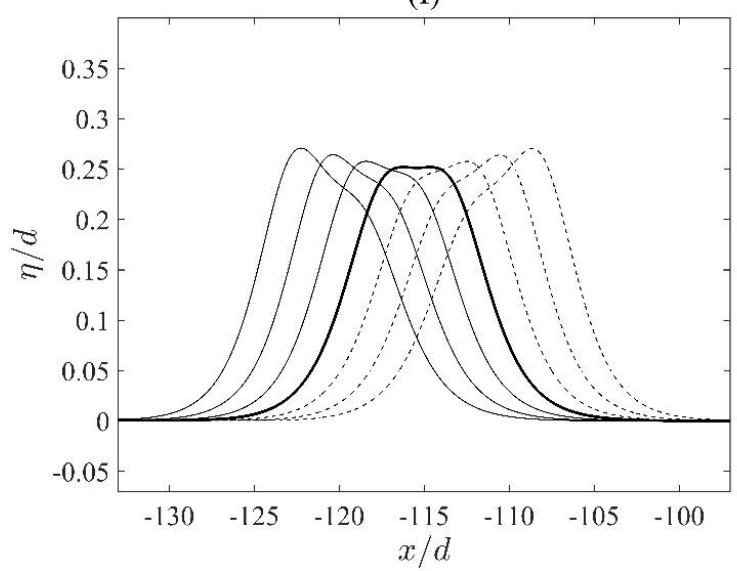


(g)

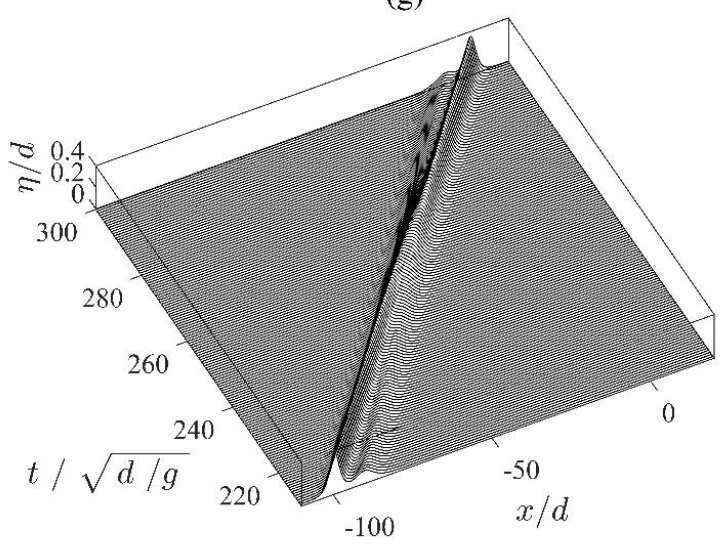

(h)

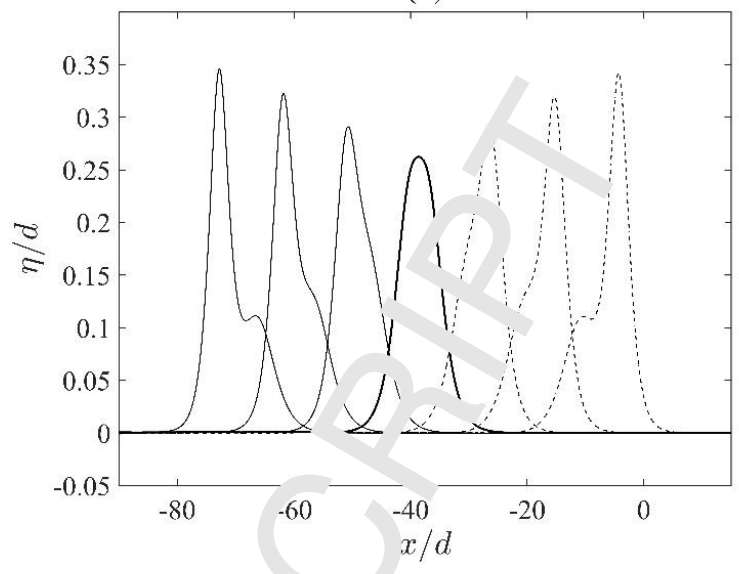

Figure 23. Overtaking collision for solitary waves of amplitudes: (a) $-r^{\prime}$, , for $\sigma_{1}=0.4$ and $\sigma_{2}=0.3$; (c)-(d) for $\sigma_{1}=0.4$ and $\sigma_{2}=0.1333$; (e)-(f) for $\sigma_{1}=0.4$ and $\sigma_{2}=0.113$ and ( $\xi^{\prime}-\left(\mathrm{h}^{\prime}\right.$ for $\sigma_{1}=0.4$ and $\sigma_{2}=0.1$. Each case shows both spatial-temporal plot (left) and wave profiles at different tiı. (righ ${ }^{\wedge}$ _ _ the critical time when two waves exchange their relative positions; __ , wave profile before the crı al time ; -........, wave profile after the critical time.

For $\sigma_{1} / \sigma_{2}=3$, shown in Figure. 23(c) (d), the higher w c calcnes up with the smaller wave and then partially absorbs it. The dual-crest wave profile in our simulation at the critica' 'ime belongs to Lax's [28] category (a) though the distance between the two crests is significantly smaller tha tha 1 in the first case with $\sigma_{1}=0.4$ and $\sigma_{2}=0.3$. But the amplitude ratio tells us that the result should have belone $t$ to L x's [28] category (b) or (c). So there is a conflict of our numerical result with the category of Lax [28].

Figure. 23(f) shows that there is a flattened peak ....n $n$. distinctive dual crests at the critical time for $\sigma_{1} / \sigma_{2}=3.539$. The wave profile is supposed to be in the form of a single c. ' ct based on category (c) of Lax [28] at the critical time, while this ratio is defined as a critical transition value fr- ategory (b) to (c) in Craig et al. [3]. Our present numerical result for this case is essentially consistent with the revis $\_$categor 'proposed by Craig et al. [3].

Figure. 23(h) shows only a single crest at th. r rit al time for the case with $\sigma_{1}=0.4$ and $\sigma_{2}=0.1$. The larger wave gradually swallows the smaller one durin, the overtaking process. After the critical time, two waves re-emerge with a change of relative positions. This behavinur, ne s' ne as category (c) both in Lax [28] and Craig et al. [3].

Table 6: Spatial phase $s_{\mathbf{s}} \cdot \mathrm{f}_{\mathrm{t}} \mathrm{s}$ and categary for overtaking collision of solitary waves

\begin{tabular}{|c|c|c|c|c|c|c|c|c|c|}
\hline Case & $\sigma_{1}$ & $\sigma_{2}$ & $\sigma_{1} / s_{2}$ & $\begin{array}{c}\text { Category in } \\
\text { Lax [28] }\end{array}$ & $\begin{array}{c}\text { Category in } \\
\text { Craig et al. }[3]\end{array}$ & $\hat{s}_{1}$ & $\hat{s}_{2}$ & $s_{1}$ & $s_{2}$ \\
\hline 1 & 0.4 & 0.3 & .1333 & $\mathrm{a}$ & $\mathrm{a}$ & 5.7688 & -6.3883 & 5.8896 & -6.8007 \\
\hline 2 & 0.4 & 0.1333 & $?$ & $\mathrm{~b}$ or $\mathrm{c}$ & $\mathrm{b}$ & 2.3666 & -5.3963 & 2.9443 & -5.1004 \\
\hline 3 & 0.4 & 0.113 & 3.5 & $\mathrm{c}$ & $\mathrm{b}$ or $\mathrm{c}$ & 2.5960 & -3.9291 & 2.6486 & -4.9832 \\
\hline 4 & 0.4 & 0.1 & . & $\mathrm{c}$ & $\mathrm{c}$ & 2.9604 & -3.834 & 2.4566 & -4.9131 \\
\hline
\end{tabular}

Table 6 gives ne thec etical and numerical result of the phase shifts as well as the category of the overtaking collision. $\hat{s}_{1}$ and $\hat{s}_{2}$ are the $\mathrm{p}$ esent nu nerical results and $s_{1}$ and $s_{2}$ represent theoretical results by Eq. (21). It can be found that the numerical results agree well with analytical data.

\section{Conclusion:}

A thorough analysis on the generation, propagation and interaction of solitary waves in a nonlinear numerical water wave tank based on the 2D Harmonic Polynomial Cell method is presented.

Four different methods of solitary wave generation are implemented in the numerical water wave tank. For $\sigma>0.4$, the 
results by Goring's method are found to be less satisfactory because of reduction in amplitude and obvious trailing waves. But the FNL method can produce solitary waves with minor trailing waves even for highly nonlinear waves.

For the head-on collision of solitary waves, our numerical results of the maximum run-up are in excellent agreement with the third-order approximation by Su \& Mirie [1] up to $\sigma=0.5$. For even higher waves, highe order nonlinearity is not negligible so that the Su \& Mirie's [1] theory fails to provide accurate prediction. But our fully -nonlıı $\neg \mathbf{r}$ numerical wave tank can still get the highly accurate results. Moreover, for the symmetric collision with $\sigma=\jmath .4$, jur numerical results of wave profiles, phase shifts and the trailing waves after the collision are also successful, va dated with experimental measurements of Chen \& Yeh [2].

The phase shifts after the head-on collision are thoroughly investigated. Many res _. hers _. ve questioned that Su \& Mirie's [1] third-order prediction consistently underestimates the phase shifts when omp ing with either their numerical results or experimental measurements. In present study, we demonstrate that the phase $\mathrm{s}_{\mathrm{s}}$ ift near to the collision center is larger than that far away from the collision domain. However, those resear hers a: vays mistakenly compared their immediate phase shifts with Su \& Mirie's [1] third-order approximation of the un. 'orm ph se shifts. This is the main reason for the discrepancy. Additionally, inspired by Cooker et al. [26], we suggest " $\mathrm{u}$ use the general 'fully interacting time' as the phase lag in time for both symmetric and asymmetric head-on collisio $F_{A}$ th theoretical expression of the fully interacting time is not obtained, which could be a valuable research in the : ture $\cdots$.

The overtaking collision of solitary waves is also studied for selected cases. Our numerical result is well validated with the numerical and experimental result of Craig et al. [3]. Furthermnre, L. - sim .lations of four selected cases also strongly support Craig et al.'s [3] category of the overtaking collision process A. for the phase shifts, our present results are also in good consistent with the analytical results by Li [52].

In the near future, we shall move our simulation into deep water wa s to study the wave-wave interaction problem.

\section{Acknowledgements}

We would like to acknowledge the National Natura, Sc. nce Foundation of China [grant number 51761135012, 51479114 and 11742021], and NSFC-RCUK_EPSRC . $1 . . . f_{n}$ financial support. This work was also partly supported by the Research Council of Norway through the Centre of Excc. 'snce funding scheme, Project number 223254-AMOS.

\section{References}

[1] C.H. Su, R.M. Mirie, On head-on cr!lisio. br.ween two solitary waves, J. Fluid Mech. 98 (1980) 509-525. doi:10.1017/S0022112080000262.

[2] Y. Chen, H. Yeh, Laboratory experimeı. on cr unter-propagating collisions of solitary waves. Part 1. Wave interactions, J. Fluid Mech. 749 (2014) 577-5c doi:10.1u17/jfm.2014.231.

[3] W. Craig, P. Guyenne, J. Hammack, D. . `onderson, C. Sulem, Solitary water wave interactions, Phys. Fluids. 18 (2006) 1-25. doi:10.1063/1.2205916

[4] J. Scott Russell, Report on v ve , in: Rep. Fourteenth Meet. Br. Assoc. Adv. Sci., 1844: pp. 311-390.

[5] J.L. Hammack, H. Segur The Kor weg-de Vries equation and water waves. Part 2. Comparison with experiments, J. Fluid Mech. 65 (1974) 89-'14. 'r ji:10.1017/S002211207400139X.

[6] T. kishi and H. Saeki the $S_{1} \cdot{ }^{\prime}$ ng, Breaking and Runup Op the Solitary Wave on Impermeable Rough Slopes, Coast. Eng. (1966) 322-3 7. doi:1 1007/978-94-6091-478-2_21.

[7] F.E. Camfield, R.L Street Shoaling of Solitary Waves on Small Slopes, J. Waterw. Harb. Div. 95 (1969) 1-22. http://cedb.ass ..org/çi/WWWdisplay.cgi?16167.

[8] D.G. Goring, Tsunam ;: the propagation of long waves onto a shelf, 1979. Phd Thesis. California Institute of Technology, Pasm ...d, CA.

[9] G. Katell, '. E' c, _ ıccuracy of solitary wave generation by a piston wave maker., J. Hydraul. Res. 40 (2002) 321-331. doi:10.1080/ '221680209499946.

[10] S. Malek-Mohammadi, F. Testik, New methodology for laboratory generation of solitary waves, J. Waterw. Port, 136 (2010) 286-294. doi:10.1061/(ASCE)WW.1943-5460.0000046.

[11] N.J. Wu, S.C. Hsiao, H.H. Chen, R.Y. Yang, The study on solitary waves generated by a piston-type wave maker, Ocean Eng. 117 (2016) 114-129. doi:10.1016/j.oceaneng.2016.03.020. 
[12] M.J. Boussinesq, Théorie de l'intumescence liquide, appelée onde solitaire ou de translation, se propageant dans un canal rectangulaire, C. R. Acad. Sci. Paris 72 (1871) 755-759

[13] C.E. Synolakis, The runup of solitary waves, J. Fluid Mech. 185 (1987) 523-545. doi:10.1017/S002211208700329X.

[14] J.D. Ramsden, Forces on a Vertical Wall due to Long Waves, Bores, and Dry-Bed Surges, Waterw. Port, Coastal, Ocean Eng. 122 (1996) 134-141. doi:10.1061/(ASCE)0733-950X(1996)122:3(134).

[15] A. Jensen, G.K. Pedersen, D.J. Wood, An experimental study of wave run-up at a steep Jea ', J. Fluid Mech. (2003) 161-188. doi:10.1017/S0022112003004543.

[16] S. Grilli, I.A. Svendsen, The propagation and runup of solitary waves on steep slope. Rep. .' '. 91-4, Ctr. for App1. Coast. Res., Univ. of Delaware, Newark, Del., 1991.

[17] L. Rayleigh, On waves, Phil. Mag. 1 (1876) 257-279.

[18] N.J. Wu, T.K. Tsay, Y.Y. Chen, Generation of stable solitary waves by a piston-ty ${ }_{r}$ wave maker, Wave Motion. 51 (2014) 240-255. doi:10.1016/j.wavemoti.2013.07.005.

[19] J. Fenton, A ninth-order solution for the solitary wave, J. Fluid Mech. 53 (19, ?) 257-' 71. doi:10.1017/S002211207200014X.

[20] J.G.B. Byatt-Smith, An integral equation for unsteady surface waves nd $\_$col ment on the Boussinesq equation, J. Fluid Mech. 49 (1971) 625-633. doi:10.1017/S0022112071002295.

[21] T. Maxworthy, Experiments on collisions between solitary waves, J. Fluid Mech. 76 (1976) 177-186. doi:10.1017/S0022112076003194.

[22] R.M. Mirie, C.H. Su, Collisions between two solitary waves. Paı ? A numerical study, J. Fluid Mech. 115 (1982) 475-492.

[23] J.D. Fenton, M.M. Rienecker, A Fourier method for solving non 'ear water wave problems: Application to solitary wave interactions, J. Fluid Mech. 118 (1982) 411-443.

[24] R.K.C. Chan, R.L. Street, A computer study of fi ‘e-am ${ }^{+}$'itude water waves, J. Comput. Phys. 6 (1970) 68-94. doi:10.1016/0021-9991(70)90005-7.

[25] D.P. Renouard, F.J.S. Santos, A.M. Temperville, in ntal study of the generation, damping, and reflexion of a solitary wave, Dyn. Atmos. Ocean. 9 (1985) 341-358. i $10.1016 / 0377-0265(85) 90008-9$.

[26] M. Cooker, P. Weidman, D. Bale, Reflectior ? a high-amplitude solitary wave at a vertical wall, J. Fluid Mech. 342 (1997) 141-158. doi:10.1017/S002211209'00551X

[27] Y. Chen, E. Zhang, H. Yeh, Laboratory expe, nen s on counter-propagating collisions of solitary waves. Part 2. Flow

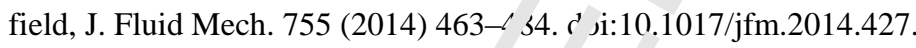

[28] P.D. Lax, Integrals of nonlinear equatı. of e slution and solitary waves, Commun. Pure Appl. Math. 21 (1968) 467490. doi:10.1002/cpa.316021050?

[29] P.D. Weidman, T. Maxworthy, Lxperı. nts on strong interactions between solitary waves, J. Fluid Mech. 85 (1978) 417-431. doi:10.1017/S0022' \& 2u'8000713.

[30] N.J. Zabusky, M.D. Kruska, 'ntf action of "solitons" in a collisionless plasma and the recurrence of initial states, Phys. Rev. Lett. 15 (1965) 240- 243. do1. ?.1103/PhysRevLett.15.240.

[31] C.S. Gardner, J.M. Gre ne, ' 1.D. Juskal, R.M. Miura, Method for solving the Korteweg-deVries equation, Phys. Rev. Lett. 19 (1967) 1095-109\% . i. 0.1103/PhysRevLett.19.1095.

[32] R.L. Sachs, Comp! teness ` `derivatives of squared Schrödinger eigenfunctions and explicit solutions of the linearized KdV equation. SIA``Journ 1 on Mathematical Analysis 14.4 (1983) 674-683.

[33] Q. Zou, C.H su, Cvertaking collision between two solitary waves., PHYS. FLUIDS. 29 (1986) $2113-2123$. doi:10.1063/1 965599.

[34] S.T. Grilli, J. Sku_ _. p, I.A. Svendsen, An efficient boundary element method for nonlinear water waves, Eng. Anal. Bound. El n. + < (1989) 97-107. doi:10.1016/0955-7997(89)90005-2.

[35] J.C. Harris, E. Dombre, M. Benoit, S.T. Grilli, Fast integral equation methods for fully nonlinear water wave modeling, in: Proc. Int. Ofrishore Polar Eng. Conf., 2014.

[36] Y.-L. Shao, O.M. Faltinsen, Towards Efficient Fully-Nonlinear Potential-Flow Solvers in Marine Hydrodynamics, in: Vol. 4 Offshore Geotech. Ronald W. Yeung Honor. Symp. Offshore Sh. Hydrodyn., 2012: p. 369. doi:10.1115/OMAE2012-83319. 
[37] Y.L. Shao, O.M. Faltinsen, A harmonic polynomial cell (HPC) method for 3D Laplace equation with application in marine hydrodynamics, J. Comput. Phys. 274 (2014) 312-332. doi:10.1016/j.jcp.2014.06.021.

[38] A.G. Fredriksen, T. Kristiansen, O.M. Faltinsen, Experimental and numerical investigation of wave resonance in moonpools at low forward speed, Appl. Ocean Res. 47 (2014) 28-46. doi:10.1016/j.apor.2014 13.005.

[39] H. Liang, O.M. Faltinsen, Y.L. Shao, Application of a 2D harmonic polynomial cell (HPC, meth ' ${ }^{1}$ to singular flows and lifting problems, Appl. Ocean Res. 53 (2015) 75-90. doi:10.1016/j.apor.2015.07.011

[40] W. Zhu, M. Greco, Y. Shao, Improved HPC method for nonlinear wave tank, Int. J. Na、 4r' ait. Ocean Eng. 9 (2017). doi:10.1016/j.ijnaoe.2017.03.009.

[41] F.C.W. Hanssen, A. Bardazzi, C. Lugni, M. Greco, Free-surface tracking in 2D . . ? the . armonic polynomial cell method: Two alternative strategies, Int. J. Numer. Methods Eng. 113 (2018) 311- 51. i ji:1u.1002/nme.5615.

[42] S. Ma, F.C.W. Hanssen, M.A. Siddiqui, M. Greco, O.M. Faltinsen, Local and s hal properties of the harmonic polynomial cell method: In-depth analysis in two dimensions, Int. J. Nu ler. Mc hods Eng. 113 (2018) 681-718. doi:10.1002/nme.5631.

[43] A. Bardazzi, C. Lugni, M. Antuono, O.M. Faltinsen, Generalized HPC atnod tor the Poisson equation. J. Comput. \& Phys, 299 (2015) 630-648. doi.org/10.1016/j.jcp.2015.07.026.

[44] J. Wang, O.M. Faltinsen, A harmonic polynomial method based on - $\urcorner$ rtesi $\cdots$ orids with local refinement for complex wave-body interactions. http://www.iwwwfb.org/workshops/33.htm/, 2018.

[45] F.-C.W. Hanssen, M. Greco, Y. Shao, The Harmonic Polynomiaı Tell cethod for Moving Bodies Immersed in a Cartesian Background Grid, in: Vol. 11 Prof. Robert F. ?eck Honor. Symp. Mar. Hydrodyn., 2015. doi:10.1115/OMAE2015-41282.

[46] M. Greco, A two-dimensional study of green-water loading, 200 . Phd Thesis. Norwegian University of Science and Technology

[47] R. Grimshaw, The solitary wave in water of v riable depth. Part 2, J. Fluid Mech. 46 (1971) 611-622. doi:10.1017/S0022112071000739.

[48] D. Clamond, D. Dutykh, Fast accurate computa. $v^{c} c^{\text {th. }}$ fully nonlinear solitary surface gravity waves, Comput. \& Fluids. 84 (2013) 35-38. doi:10.1016/j.compfluid.201د. ^5.010.

[49] G. He, M. Kashiwagi, Numerical analysis of hydroelastic behavior of a vertical plate due to solitary waves, J. Mar. Sci. Technol. (2012). doi:10.1007/s00773-' 11-0155 7.

[50] R.C. Ertekin, M. Hayatdavoodi, J.W Kiıs, Or some solitary and cnoidal wave diffraction solutions of the Green-Naghdi equations, Appl. Ocear Res. 2014). doi:10.1016/j.apor.2014.04.005.

[51] M. Tanaka, The stability of solitary wav Phr .. Fluids. 29 (1986) 650-655. doi:10.1063/1.865459.

[52] W. Li, Amplification of Solitary V 'ves along a Vertical Wall, 2012. Phd Thesis. Oregon State University 